\title{
Implications from neurologic assessment of brain protection for total arch replacement from a randomized trial
}

\author{
Lars G. Svensson, MD, PhD, ${ }^{\mathrm{a}, \mathrm{b}}$ Eugene H. Blackstone, MD, ${ }^{\mathrm{a}, \mathrm{b}, \mathrm{c}}$ Carolyn Apperson-Hansen, MStat, ${ }^{\mathrm{d}}$ \\ Paul M. Ruggieri, MD, ${ }^{\mathrm{e}}$ Ponnuthurai Ainkaran, MS, ${ }^{\mathrm{c}}$ Richard I. Naugle, PhD, ${ }^{\mathrm{f}}$ Brian Lima, MD, \\ Eric E. Roselli, MD, ${ }^{\mathrm{a}, \mathrm{b}}$ Maxwell Cooper, BS, ${ }^{\mathrm{b}}$ David Somogyi, CCP, ${ }^{\mathrm{g}}$ E. Murat Tuzcu, MD, ${ }^{\mathrm{h}}$ \\ Samir Kapadia, MD, ${ }^{\mathrm{h}}$ Daniel G. Clair, MD, ${ }^{\mathrm{a}, \mathrm{i}}$ Joseph F. Sabik III, MD, ${ }^{\mathrm{a}, \mathrm{b}}$ and Bruce W. Lytle, MD ${ }^{\mathrm{a}, \mathrm{b}}$
}

\section{ABSTRACT}

Objective: The study objective was to perform a randomized trial of brain protection during total aortic arch replacement and identify the best way to assess brain injury.

Methods: From June 2003 to January 2010, 121 evaluable patients were randomized to retrograde $(n=60)$ or antegrade $(n=61)$ brain perfusion during hypothermic circulatory arrest. We assessed the sensitivity of clinical neurologic evaluation, brain imaging, and neurocognitive testing performed preoperatively and 4 to 6 months postoperatively to detect brain injury.

Results: A total of 29 patients $(24 \%)$ experienced neurologic events. Clinical stroke was evident in 1 patient $(0.8 \%)$, and visual changes were evident in 2 patients; all had brain imaging changes. A total of 14 of 95 patients $(15 \%)$ undergoing both preoperative and postoperative brain imaging had evidence of new white or gray matter changes; 10 of the 14 patients had neurocognitive testing, but only 2 patients experienced decline. A total of 17 of 96 patients (18\%) undergoing both preoperative and postoperative neurocognitive testing manifested declines of 2 or more reliable change indexes; of these 17, 11 had neither imaging changes nor clinical events. Thirty-day mortality was $0.8 \%$ $(1 / 121)$, with no neurologic deaths and a similar prevalence of neurologic events after retrograde and antegrade brain perfusion $(22 / 60,37 \%$ and $15 / 61,25 \%$, respectively; $P=.2$ ).

Conclusions: Although this randomized clinical trial revealed similar neurologic outcomes after retrograde or antegrade brain perfusion for total aortic arch replacement, clinical examination for postprocedural neurologic events is insensitive, brain imaging detects more events, and neurocognitive testing detects even more. Future neurologic assessments for cardiovascular procedures should

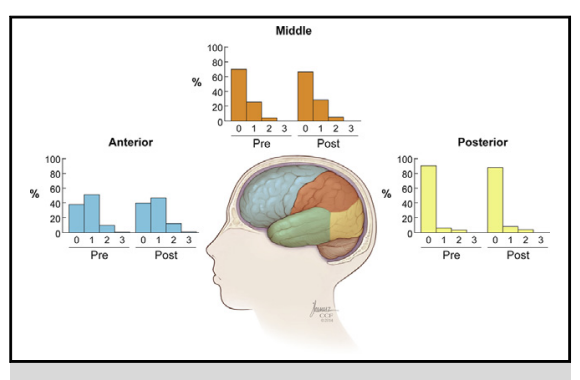

Brain imaging changes preoperatively and 4 to 6 months postoperatively according to location.

\section{Central Message}

Future neurologic assessments for cardiovascular procedures should include brain imaging studies and neurocognitive testing.

\section{Perspective}

Although this randomized trial revealed similar neurologic outcomes after RBP or ABP for total aortic arch replacement during hypothermic circulatory arrest, clinical examination for postprocedural events is insensitive, brain imaging detects more events, and neurocognitive testing detects even more. Future studies should include all these neurologic assessment modalities.

See Editorial Commentary page 1148. include not only clinical examination but also brain imaging studies, neurocognitive testing, and long-term assessment. (J Thorac Cardiovasc Surg 2015;150:1140-7)

\footnotetext{
From the ${ }^{\mathrm{a} A o r t i c}$ Center, Cleveland Clinic, Cleveland, Ohio; Departments of ${ }^{\mathrm{b}}$ Thoracic and Cardiovascular Surgery and ${ }^{\mathrm{C}}$ Quantitative Health Sciences, Cleveland Clinic, Cleveland, Ohio; ${ }^{\mathrm{d}}$ School of Medicine, Case Western Reserve University, Cleveland, Ohio; Departments of ${ }^{\mathrm{e}}$ Neuroradiology, ${ }^{\mathrm{f}}$ Psychiatry and Psychology, ${ }^{\mathrm{g}}$ Perfusion Services, ${ }^{\mathrm{h}}$ Cardiovascular Medicine and ${ }^{\mathrm{i}}$ Vascular Surgery, Cleveland Clinic, Cleveland, Ohio.

This study was supported in part by the Gus P. Karos Family Registry Fund, the David Whitmire Hearst, Jr Foundation, the Sheikh Hamdan bin Rashid Al Maktoum Distinguished Chair in Thoracic and Cardiovascular Surgery (held by J.F.S.), and the Kenneth Gee and Paula Shaw, PhD, Chair in Heart Research (held by E.H.B.).
}

ClinicalTrials.gov Identifier: NCT00606190.

Received for publication Oct 9, 2014; revisions received July 16, 2015; accepted for publication July 20, 2015; available ahead of print Sept 22, 2015.

Address for reprints: Lars G. Svensson, MD, PhD, Aortic Center and Heart and Vascular Institute, Cleveland Clinic, 9500 Euclid Ave/Desk J4-1, Cleveland, OH 44915 (E-mail: svenssl@ccf.org).

$0022-5223 / \$ 36.00$

Copyright (C) 2015 Published by Elsevier Inc. on behalf of The American Association for Thoracic Surgery

http://dx.doi.org/10.1016/j.jtcvs.2015.07.054 


\section{Abbreviations and Acronyms}

$\mathrm{ABP}=$ antegrade brain perfusion

$\mathrm{CT}=$ computed tomography

EEG = electroencephalography

MRI = magnetic resonance imaging

$\mathrm{RBP}=$ retrograde brain perfusion

$\mathrm{TAVR}=$ transcatheter aortic valve replacement

Supplemental material is available online.

Among cardiovascular procedures, percutaneous transcatheter aortic valve replacement (TAVR) and aortic arch replacement are considered to present the highest risk for postprocedure neurologic events. ${ }^{1-15}$ In the Placement of AoRTic TraNscathetER (PARTNER) valve trials, risks of clinical stroke or neurocognitive deficits were $4.6 \%$ to $6.4 \%$ for transfemoral TAVR. ${ }^{16,17}$ However, other studies have reported strokes detected by postprocedure diffusion-weighted magnetic resonance imaging (MRI) in $68 \%$ to $84 \%$ of these patients. ${ }^{1,2,4}$ In a large retrospective study of 656 patients undergoing aortic arch replacement without adjunctive brain perfusion, the risk of clinically detectable strokes was 7\%. ${ }^{18}$ A subsequent prospective study using brain perfusion showed a $2.8 \%$ risk of stroke, but 2 to 3 weeks after surgery, $9 \%$ of patients had new neurocognitive deficits. ${ }^{14}$ An investigation of 142 patients undergoing total arch replacement with preoperative brain MRI studies using the Scheltens scale for leukoaraiosis showed that residual focal neurologic deficits developed in $4.2 \%$, and total white matter scores were strongly correlated with type 2 nonfocal neurocognitive changes. ${ }^{10}$ Internet-based assessment of postoperative neurocognitive function has shown that longer duration of hypothermic circulatory arrest for these operations is associated with slow processing speed and low memory scores. ${ }^{19}$

Although clinical stroke risk may seem to be acceptable for both TAVR and total arch replacement, it is likely that MRI-detected strokes occur more frequently, and it is unclear how these MRI lesions are associated with neurocognitive deficits. Because of this uncertainty about (1) the importance of clinical detection of neurologic events after cardiovascular procedures, (2) the changes noted on MRI and their potential neurocognitive consequences, and (3) whether adjunctive measures protect the brain, we have explored in depth the neurologic findings from a randomized study of brain-protection strategies during aortic arch repair to discover their implications for future studies of neurologic consequences of interventions such as TAVR and aortic arch procedures that may dislodge aortic plaque material.

\section{MATERIALS AND METHODS \\ Study Design}

This study is based on neurologic outcomes data from a randomized single-blinded 1:1 trial of antegrade brain perfusion (ABP) versus retrograde brain perfusion ( $\mathrm{RBP}$ ) during hypothermic circulatory arrest in patients undergoing total aortic arch replacement. The trial's individual neurologic end points were (1) clinical neurologic events, (2) brain imaging changes, and (3) reliable change index on neurocognitive testing. The complete randomized trial protocol, with details of inclusion and exclusion criteria and sample size considerations, is presented in Appendix E1.

\section{Patients}

From June 2003 to January 2010, 341 patients underwent total aortic arch operations at Cleveland Clinic, of whom 122 met inclusion and exclusion criteria and provided written consent to participate in the trial (CONSORT diagram, Figure E1). One patient died after randomization but before surgery, leaving 121 evaluable patients, 60 randomized to $\mathrm{RBP}$ and 61 randomized to ABP. Mean age overall was $58 \pm 12$ years, $48(40 \%)$ were women, $16(13 \%)$ had a history of stroke, and $52(43 \%)$ had a history of carotid disease, following definitions for the Society of Thoracic Surgeons National Database (http://www.sts.org/sites/default/ files/documents/STSAdultCVDataSpecificationsV2_81.pdf). Eleven patients $(9 \%)$ had peripheral arterial disease in addition to their aortic disease (Table 1). Forty-seven patients $(39 \%)$ had undergone previous cardiac surgery.

\section{Surgical Context and Brain Perfusion}

All 121 patients underwent total arch replacement; 74 procedures $(61 \%)$ were done using the elephant trunk procedure. ${ }^{12}$ Replacement of the total aortic arch was performed using right subclavian arterial inflow, cooling to an esophageal temperature of less than $20^{\circ} \mathrm{C}$ using alpha-stat strategy, ${ }^{11,12,14}$ circulatory arrest with the patient's head down and packed in ice, and $\mathrm{CO}_{2}$ field flooding. ${ }^{14}$ Bispectral monitoring of anesthesia depth was routine, but not continuous electroencephalography. RBP was done by perfusing the distal superior vena cava with proximal occlusion above the azygos vein; perfusion was continuous during the period of circulatory arrest, with pressure monitoring so as not to exceed 25 to $30 \mathrm{~cm} \mathrm{H}_{2} \mathrm{O}{ }^{18} \mathrm{ABP}$, at a pressure of 45 to $60 \mathrm{~mm} \mathrm{Hg}$, involved perfusing the brachiocephalic and carotid arteries for 5 minutes approximately 10 to 15 minutes after instituting circulatory arrest using retrograde cardioplegia balloon catheters and, if needed, perfusing again after a further 15 minutes. L.G.S. performed all operations except for 3 patients.

Patients were rewarmed to more than $36^{\circ} \mathrm{C}$ venous temperature and weaned from cardiopulmonary bypass. Mean cardiopulmonary bypass time was $118 \pm 33$ minutes, circulatory arrest time was $27 \pm 13$ minutes, and myocardial ischemic time was $67 \pm 35$ minutes (Table E1). Postoperatively, all patients were placed on chronic aspirin therapy, $162 \mathrm{mg}$ daily, and statins were prescribed for all with coronary artery disease or elevated lipids.

\section{End Points}

The trial's primary end point was a composite of (1) hospital death from neurologic causes, (2) postoperative clinical stroke, (3) brain imaging changes, and (4) reliable neurocognitive decline.

Clinical neurologic events. Clinical neurologic events were recorded in-hospital after the index surgery. They included stroke, visual 
TABLE 1. Patient characteristics overall and by brain perfusion method

\begin{tabular}{|c|c|c|c|c|c|c|}
\hline \multirow[b]{3}{*}{ Variable } & \multirow{2}{*}{\multicolumn{2}{|c|}{ Overall $(\mathbf{n}=121)$}} & \multicolumn{4}{|c|}{ Brain perfusion method } \\
\hline & & & \multicolumn{2}{|r|}{ Retrograde $(n=60)$} & \multicolumn{2}{|r|}{ Antegrade $(n=61)$} \\
\hline & n* & No. $(\%)$ or mean \pm SD & $\mathbf{n}^{*}$ & No. $(\%)$ or mean \pm SD & n* & No. $(\%)$ or mean \pm SD \\
\hline \multicolumn{7}{|l|}{ Demographics } \\
\hline Age (y) & 121 & $58 \pm 12$ & 60 & $58 \pm 12$ & 61 & $58 \pm 13$ \\
\hline Female & 121 & $48(40)$ & 60 & $27(45)$ & 61 & $21(34)$ \\
\hline Body mass index $\left(\mathrm{kg} \cdot \mathrm{m}^{-2}\right)$ & 118 & $28 \pm 6.4$ & 58 & $29 \pm 7.8$ & 60 & $28 \pm 4.5$ \\
\hline \multicolumn{7}{|l|}{ Preoperative neurologic morbidity } \\
\hline Prior stroke & 121 & $16(13)$ & 60 & $10(17)$ & 61 & $6(9.8)$ \\
\hline History of carotid disease & 121 & $52(43)$ & 60 & $26(43)$ & 61 & $26(43)$ \\
\hline \multicolumn{7}{|l|}{ Cardiovascular comorbidity } \\
\hline Peripheral arterial disease & 121 & $11(9.1)$ & 60 & $4(6.7)$ & 61 & $7(11)$ \\
\hline Hypertension & 121 & $98(81)$ & 60 & $51(85)$ & 61 & $47(77)$ \\
\hline Preoperative atrial fibrillation or flutter & 119 & $2(1.7)$ & 59 & $1(1.7)$ & 60 & $1(1.7)$ \\
\hline \multicolumn{7}{|l|}{ Aortic valve disease } \\
\hline Stenosis & 121 & $10(8.3)$ & 60 & $4(6.7)$ & 61 & $6(9.8)$ \\
\hline Regurgitation & 121 & $47(39)$ & 60 & $22(37)$ & 61 & $25(41)$ \\
\hline Previous cardiac operation & 121 & $47(39)$ & 60 & $24(40)$ & 61 & $23(38)$ \\
\hline CABG & 121 & $21(17)$ & 60 & $12(20)$ & 61 & $9(15)$ \\
\hline \multicolumn{7}{|l|}{ Aortic valve procedure } \\
\hline Repair & 121 & $14(12)$ & 60 & $6(10)$ & 61 & $8(13)$ \\
\hline Replacement & 121 & $43(36)$ & 60 & $23(38)$ & 61 & $20(33)$ \\
\hline \multicolumn{7}{|l|}{ Aortic valve disease } \\
\hline Stenosis & 121 & $10(8.3)$ & 60 & $4(6.7)$ & 61 & $6(9.8)$ \\
\hline Regurgitation & 121 & $47(39)$ & 60 & $22(37)$ & 61 & $25(41)$ \\
\hline \multicolumn{7}{|l|}{ Noncardiovascular comorbidity } \\
\hline Pharmacologically treated diabetes & 120 & $6(5.0)$ & 59 & $6(10)$ & 61 & $0(0)$ \\
\hline COPD & 121 & $24(20)$ & 60 & $12(20)$ & 61 & $12(20)$ \\
\hline Smoking & 120 & $73(61)$ & 59 & $37(63)$ & 61 & $37(63)$ \\
\hline Renal failure requiring dialysis & 121 & $5(4.1)$ & 60 & $2(3.3)$ & 61 & $2(3.3)$ \\
\hline
\end{tabular}

$C A B G$, Coronary artery bypass grafting; $C O P D$, chronic obstructive pulmonary disease; $S D$, standard deviation. *Patients with data available.

changes, delirium, seizures, and peripheral twitching. Whenever there was even a slight clinical suspicion that one of these might be occurring, a neurologic consultation was obtained that typically included brain imaging. Workup of seizure activity and peripheral twitching included electroencephalography (EEG).

Brain imaging. Preoperatively, 113 of the 121 patients $(93 \%)$ underwent brain imaging studies, 56 in the RBP group and 57 in the ABP group. Brain MRI was performed using a $1.5 \mathrm{~T}$ conventional clinical scanner. This preoperative study was essential for interpreting the 4- to 6-month postoperative study, particularly given the history of stroke in a substantial number of patients. Standard anatomic imaging of the brain included sagittal TSE T1, axial TSE T2, axial TSE T2-weighted fluid-attenuated inversion recovery, axial diffusion, and axial 2-dimensional gradient echocardiography or susceptibility-weighted imaging acquisitions. ${ }^{20}$ In 10 patients for whom MRI was contraindicated, a noncontrast head computed tomography (CT) scan was performed in place of the brain MRI using a 16- or 64-slice multidetector CT scanner, producing 5-mm slices in a sequential fashion with $120 \mathrm{kV}$ and 260 to $300 \mathrm{~mA}$.

Of the 113 patients, 95 were alive and underwent postoperative brain imaging 4 to 6 months postoperatively, 44 in the RBP group and 51 in the ABP group. Testing could not be performed in 4 patients who died before the scheduled tests, at an average of $5.9 \pm 1.9$ months from surgery.

All digital images were independently assessed by a neuroradiologist who was blinded to clinical history, method of surgical intervention, and results of clinical and neurocognitive testing. Each study was evaluated for the presence of major vascular territory cortical infarcts. The extent and severity of lacunar infarcts in deep gray matter structures and microvascular ischemic disease in deep white matter were each quantified on a scale of 0 to 3 using the method proposed by Wahlund and colleagues, ${ }^{21}$ which is attractive because it can be applied to MRI or CT imaging studies. Involvement of the frontal, middle (temporal and parietal), and posterior (occipital and posterior fossa) brain was scored separately to correlate with clinical evaluation and neurocognitive testing.

Neurocognitive studies. Patients were administered the same neurocognitive tests before $(n=120 ; 60$ in the RBP and 60 in the $\mathrm{ABP}$ group) and 4 to 6 months after the index procedure $(\mathrm{n}=100$; 49 in the RBP and 51 in the ABP group; average interval from surgery $=5.5 \pm 2.0$ months) by a psychometrist trained and experienced in test administration and scoring and blinded to clinical history, method of surgical intervention, and results of clinical and brain imaging studies, except that the Wide Range Achievement Test (4th ed) ${ }^{22}$ Reading subtest was administered only at baseline. The neurocognitive test battery was intentionally designed to be brief $(\sim 30$ minutes to administer), but to assess those domains that are considered most vulnerable to microvascular changes: learning and memory, timed word fluency, confrontation naming, timed manual dexterity, timed visuomotor coordination, and timed visual scanning/sequencing. These cognitive domains were assessed using the same battery that was used in earlier work investigating the effectiveness of a potential neuroprotectant in limiting cerebrovascular disease resulting from cardiac 
surgery (Appendix E2). ${ }^{23}$ Because of its brevity, this test battery's assessment of executive function was limited to set-shifting. Subjects were also asked about symptoms of depression.

For each neurocognitive test, raw scores were converted to standard scores to control for the confounding effect of age and education. ${ }^{24}$ The baseline and follow-up data were used to calculate a test-retest reliability coefficient (Pearson correlation) between preoperative and postoperative scores, a reliable change index, and associated $90 \%$ confidence interval for the change index (Appendix E1 shows details). ${ }^{25}$ Patients were considered to have had a neurocognitive decline if they had 2 or more reliable change indexes that decreased below the lower $90 \%$ confidence limit for the respective corrected reliable change index interval (Table E2). ${ }^{25}$

\section{Data Analysis}

Details of methods for neurocognitive evaluation are provided in Appendix E1. They included adjusting for age, educational level, and test-retest correlation.

Because not every patient had brain imaging and neurocognitive studies preoperatively and 4 to 6 months postoperatively, and not all patients could complete every part of the neurocognitive inventory, for patients found not to have a neurologic event, the primary end point contained a certain amount of missing data. Thus, 10-fold multiple imputation $^{26}$ was used to impute the missing values (SAS PROC MI; SAS Institute Inc, Cary, NC). Patient, procedure (preoperative and intraoperative), and brain and neurocognitive component variables (but not the composite event) were included in the multiple imputation model. Imputed end point components were dichotomized on the basis of a cutoff criterion of .5. Finally, imputed individual end point components and the composite event were tested for significance between the 2 brain perfusion methods using logistic regression (SAS PROC LOGISTIC), and inference was obtained by combining results from 10 imputed data sets with SAS PROC MIANALYZE. In a sensitivity analysis, we repeated the entire analysis process using liberal $(0.25)$ and conservative (0.75) cutoff criteria. Additional details are provided in Appendix E3.

Multivariable logistic regression analysis was used to identify variables associated with the primary end point. Variable selection involved generating 1000 bootstrap resampled data sets followed by aggregating the results of automated stepwise regression analyses. ${ }^{27,28}$

Descriptive comparisons were made among clinical neurologic assessments, brain imaging changes, and neurocognitive changes. Categoric data are summarized by frequency and percentage, with comparisons made using the chi-square test, or Fisher's exact test when frequency was less than 5 . Continuous variables are summarized by mean and standard deviation, or by 15 th, 50 th, and 85 th percentiles when values are skewed; comparisons were made using the Wilcoxon rank-sum test. Statistical analysis was conducted using SAS version 9.2 (SAS Institute Inc).

\section{RESULTS \\ Neurologic Outcomes}

There was one 30-day postoperative death $(0.8 \%)$ unrelated to a neurologic event. One patient manifested a clinically evident stroke $(0.8 \%)$, and 2 patients had visual changes. One patient experienced a seizure, 3 patients experienced peripheral twitching, and 2 patients experienced delirium. Fourteen of 95 patients $(15 \%)$ who underwent both preoperative and postoperative brain MRI or CT imaging 4 to 6 months after surgery had evidence of new infarcts or brain tissue changes on brain imaging (Table 2, Figure 1). Based on 2 or more reliable change indexes, 17 of 96 patients $(18 \%)$ who underwent both preoperative and postoperative neurocognitive testing manifested declines (Tables 2 and 3, with details in Tables E3 and E4). In total, 29 patients (24\%) experienced clinical neurologic events, cortical infarcts or deep white or gray matter changes on brain imaging, or neurocognitive changes.

Of the MRI/CT stroke events, $3(5.0 \%)$ occurred in the group of 60 patients with RBP and none occurred in the group of 61 patients with $\mathrm{ABP}(P=.12)$; however, no difference was seen in postoperative scores on brain scans for the anterior, middle, or posterior brain, and there were no statistically significant differences on neurocognitive testing except that the Boston Naming Test total score was $56.3 \pm 4.08$ for RBP and $54.1 \pm$ 7.5 for $\operatorname{ABP}(P=.04)$ and was of little clinical significance.

The primary study composite neurologic end point, obtained by 10 -fold multiple imputation of individual components, occurred in 22 of the 60 patients undergoing $\mathrm{RBP}$ and 15 of the 61 patients undergoing $\mathrm{ABP}(P=.2)$.

TABLE 2. In-hospital clinical neurologic events and postoperative brain imaging study and neurocognitive decline results

\begin{tabular}{|c|c|c|c|c|c|c|c|}
\hline \multirow[b]{3}{*}{ Event } & \multirow{2}{*}{\multicolumn{2}{|c|}{ Overall $(n=121)$}} & \multicolumn{5}{|c|}{ Brain perfusion method } \\
\hline & & & \multicolumn{2}{|c|}{ Retrograde $(n=60)$} & \multicolumn{2}{|c|}{ Antegrade $(n=61)$} & \multirow[b]{2}{*}{$P$} \\
\hline & $\mathbf{n}^{*}$ & No. $(\%)$ & $\mathbf{n}^{*}$ & No. $(\%)$ & $\mathbf{n}^{*}$ & No. $(\%)$ & \\
\hline Death due to neurologic event & 121 & $0(0)$ & 60 & $0(0)$ & 61 & $0(0)$ & 1.0 \\
\hline Clinical stroke & 121 & $1(0.83)$ & 60 & $1(1.7)$ & 61 & $0(0)$ & .5 \\
\hline Visual CNS symptoms & 121 & $2(1.7)$ & 60 & $2(3.3)$ & 61 & $0(0)$ & .2 \\
\hline Peripheral twitching & 121 & $3(2.5)$ & 60 & $0(0)$ & 61 & $3(4.9)$ & .2 \\
\hline Seizure & 121 & $1(0.83)$ & 60 & $0(0)$ & 61 & $1(1.6)$ & $>.9$ \\
\hline Delirium & 121 & $2(1.7)$ & 60 & $1(1.7)$ & 61 & $1(1.6)$ & $>.9$ \\
\hline Brain imaging changes & 95 & $14(15)$ & 44 & $9(20)$ & 51 & $5(9.8)$ & .14 \\
\hline Neurocognitive decline & $96 \dagger$ & $17(18)$ & 46 & $10(22)$ & 50 & $7(14)$ & .3 \\
\hline Composite event & 90 & $29(32)$ & 43 & $17(40)$ & 47 & $12(26)$ & .16 \\
\hline
\end{tabular}

CNS, Central nervous system. *Patients with data available. †Minimum number of patients with all tests (see Table 3 ). 


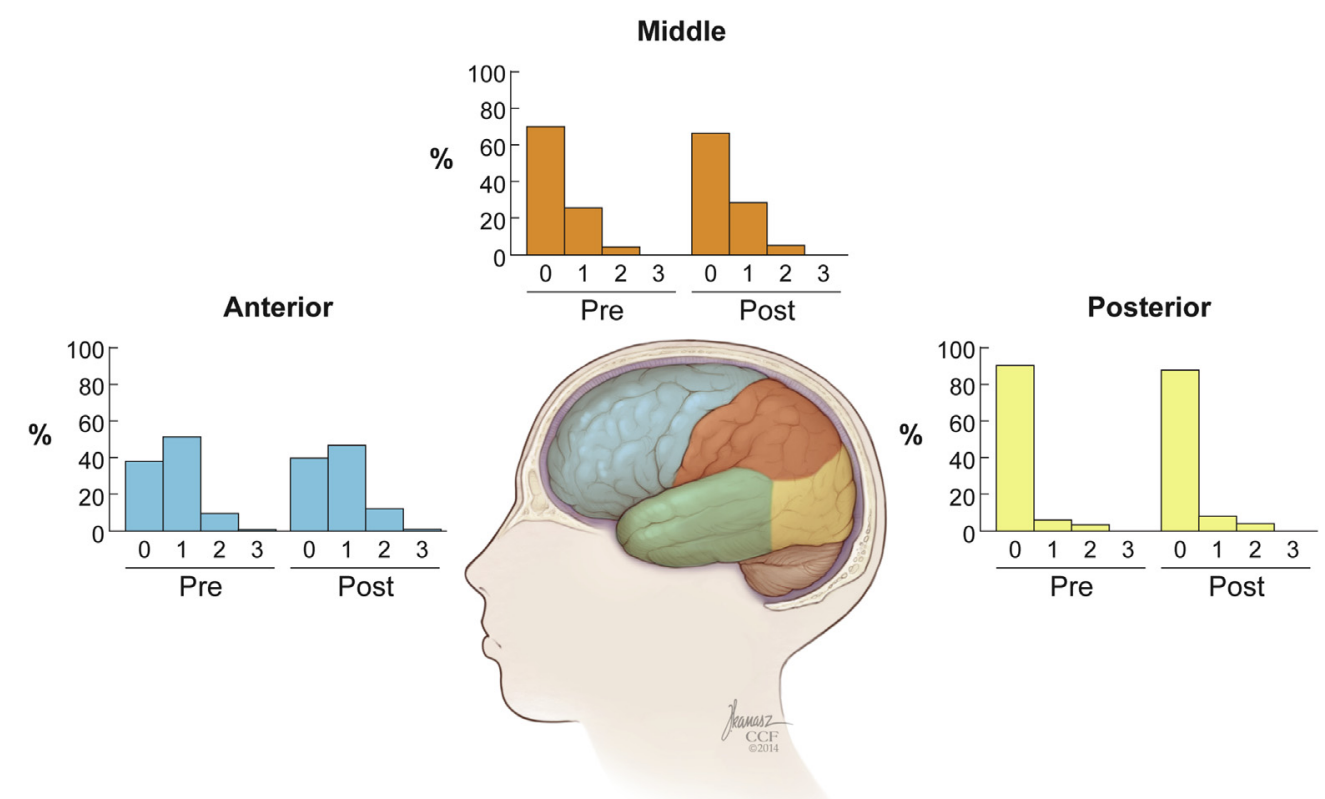

FIGURE 1. Brain imaging changes preoperatively and 4 to 6 months postoperatively according to location.

This was true whether or not a patient had experienced a preoperative stroke (Table E5). Although in general, individual neurocognitive testing revealed few changes preoperatively to postoperatively, particularly in the ABP group, postoperative scores improved for nondominant hand Grooved Pegboard and Digit Symbol tests (Tables 3, $\mathrm{E} 3$, and E4).

\section{Correlation Among Neurologic Assessment Modalities}

Occurrence of delirium, seizure, and peripheral twitching was not associated with clinical sequelae, EEG changes, or brain imaging changes, although 1 patient with visual changes experienced neurocognitive changes (Tables 4, E6, and E7). In contrast, all 3 patients experiencing a

TABLE 3. Neurocognitive test results expressed as $z$-value and reliable change

\begin{tabular}{|c|c|c|c|c|c|c|c|c|c|}
\hline \multirow[b]{2}{*}{ Test } & \multicolumn{2}{|c|}{ Preoperative result } & \multicolumn{2}{|c|}{ Postoperative result } & \multirow[b]{2}{*}{$\begin{array}{c}\begin{array}{c}\text { No. of } \\
\text { paired tests }\end{array} \\
\end{array}$} & \multirow[b]{2}{*}{$P$} & \multicolumn{3}{|c|}{ Reliable change No. $(\%)$} \\
\hline & n* & $\begin{array}{c}\text { Mean } \pm \text { SD or } \\
\text { 15th/50th/85th } \\
\text { percentiles }\end{array}$ & n* & $\begin{array}{c}\text { Mean } \pm \text { SD or } \\
\text { 15th } / 50 \text { th } / 85 \text { th } \\
\text { percentiles }\end{array}$ & & & Decline & No change & Improvement \\
\hline \multicolumn{10}{|c|}{ Hopkins Verbal Learning Test ${ }^{\dagger}$} \\
\hline Trial 1 & 120 & $1.04 \pm 0.68$ & 99 & $0.98 \pm 0.61$ & 99 & 6 & $3(3.0)$ & $92(93)$ & $4(4.0)$ \\
\hline Total & 120 & $0.32 / 1.3 / 2.3$ & 99 & $0.22 / 1.0 / 2.2$ & 99 & .2 & $7(7.1)$ & $89(90)$ & $3(3.0)$ \\
\hline Delayed recall & 120 & $0.18 / 1.0 / 2.5$ & 99 & $0.18 / 0.96 / 2.5$ & 99 & $>.9$ & $5(5.1)$ & $89(90)$ & $5(5.1)$ \\
\hline Discrimination index & 119 & $0.18 / 0.80 / 2.0$ & 99 & $0.18 / 0.73 / 2.0$ & 98 & .8 & $3(3.1)$ & $93(95)$ & $2(2.0)$ \\
\hline COWA & 118 & $0.92 \pm 0.61$ & 97 & $0.92 \pm 0.67$ & 95 & 6 & $6(6.3)$ & $82(86)$ & $7(7.4)$ \\
\hline Boston Naming Test & 119 & $-0.85 / 0.29 / 0.97$ & 97 & $-0.62 / 0.52 / 0.97$ & 96 & .5 & $4(4.2)$ & $89(93)$ & $3(3.1)$ \\
\hline \multicolumn{10}{|l|}{ Grooved Pegboard Test } \\
\hline Dominant hand & 120 & $0.98 \pm 0.76$ & 98 & $1.08 \pm 0.79$ & 98 & .5 & $4(4.1)$ & $86(88)$ & $8(8.2)$ \\
\hline Nondominant hand & 116 & $0.76 \pm 0.61$ & 97 & $0.99 \pm 0.67$ & 94 & .009 & $7(7.4)$ & $80(85)$ & $7(7.4)$ \\
\hline Digit Symbol Test & 120 & $0.69 \pm 0.54$ & 100 & $0.78 \pm 0.58$ & 100 & .02 & $4(4.0)$ & $90(90)$ & $6(6.0)$ \\
\hline Symbol search & 119 & $0.66 \pm 0.63$ & 100 & $0.77 \pm 0.60$ & 100 & .08 & $5(5.0)$ & $90(90)$ & $5(5.0)$ \\
\hline Processing speed index & 119 & $0.74 \pm 0.56$ & 100 & $0.83 \pm 0.56$ & 100 & .02 & $3(3.0)$ & $90(90)$ & $7(7.0)$ \\
\hline \multicolumn{10}{|l|}{ Trail Making Test } \\
\hline Form A & 120 & $0.83 \pm 0.58$ & 100 & $0.82 \pm 0.67$ & 100 & .8 & $5(5.0)$ & $86(86)$ & $9(9.0)$ \\
\hline Form B & 119 & $0.87 \pm 0.70$ & 99 & $0.96 \pm 0.71$ & 98 & .5 & $5(5.1)$ & $89(91)$ & $4(4.1)$ \\
\hline
\end{tabular}

COWA, Controlled Oral Word Association Test; $S D$, standard deviation. *Patients with data available. $\dagger$ Revised. 
TABLE 4. Matrix of postoperative clinical events, brain imaging, and neurocognitive outcomes

\begin{tabular}{|c|c|c|c|c|c|c|c|c|}
\hline \multirow[b]{2}{*}{ Pattern } & \multirow[b]{2}{*}{ No. of patients } & \multicolumn{5}{|c|}{ Clinical } & \multirow[b]{2}{*}{ Brain imaging changes } & \multirow[b]{2}{*}{ Neurocognitive decline } \\
\hline & & Stroke & Visual & Seizure & Twitching & Delirium & & \\
\hline 1 & 1 & Yes & No & No & No & No & Yes & N/A \\
\hline 2 & 1 & No & Yes & No & No & No & Yes & No \\
\hline 3 & 1 & No & Yes & No & No & No & Yes & Yes \\
\hline 4 & 1 & No & No & Yes & No & No & No & No \\
\hline 5 & 1 & No & No & No & Yes & No & No & N/A \\
\hline 6 & 2 & No & No & No & Yes & No & No & No \\
\hline 7 & 1 & No & No & No & No & Yes & N/A & N/A \\
\hline 8 & 1 & No & No & No & No & Yes & No & No \\
\hline 9 & 11 & No & No & No & No & No & N/A & N/A \\
\hline 10 & 10 & No & No & No & No & No & N/A & No \\
\hline 11 & 4 & No & No & No & No & No & N/A & Yes \\
\hline 12 & 8 & No & No & No & No & No & No & N/A \\
\hline 13 & 3 & No & No & No & No & No & Yes & N/A \\
\hline 14 & 7 & No & No & No & No & No & Yes & No \\
\hline 15 & 1 & No & No & No & No & No & Yes & Yes \\
\hline 16 & 57 & No & No & No & No & No & No & No \\
\hline 17 & 11 & No & No & No & No & No & No & Yes \\
\hline
\end{tabular}

N/A, Not available.

clinical stroke or visual changes exhibited new brain imaging changes. Of the 2 patients with visual changes, both had occipital imaging changes; 1 also had reliable neurocognitive decline. One patient experiencing a clinical stroke could not be tested for neurocognitive change.

Ten of the 14 patients with brain imaging changes underwent neurologic testing. Two had neurocognitive decline, and of the remaining 8 patients without neurocognitive decline, 3 had posterior occipital or cerebellar imaging changes and 1 had evidence of progressive microvascular disease.

Among the 17 patients with 2 or more reliable change indexes indicative of neurologic decline, 2 had evidence of infarcts on brain imaging and 11 had no clinical neurologic events or brain imaging changes. Thus, not all MRI/ CT changes were associated with neurocognitive decline, and not all neurocognitive declines were associated with an MRI/CT event $(P=.8)$.

We were unable to detect any reliable predictor of the primary composite end point. Specifically, the frequency of postoperative new-onset atrial fibrillation was similar between brain perfusion groups (Table E8).

\section{DISCUSSION}

\section{Principal Findings}

In this randomized trial of RBP versus $\mathrm{ABP}$ during circulatory arrest for total aortic arch replacement, clinical neurologic events, brain imaging studies, neurocognitive testing, and the composite end point failed to show that ABP offers better protection than RBP. Our study may have been underpowered to detect differences because, in our power calculations before the trial, we anticipated more events on the basis of published data available at the time the study was designed. These failed to materialize, and our results were considerably better than expected.

\section{Neurologic Outcomes in Context}

This study has shown that low clinical stroke risk can be achieved with total aortic arch replacement; however, as noted also for TAVR, ${ }^{1,2,4}$ occurrence of brain imaging events was higher than clinical neurologic events, and in this study, neurocognitive changes were even more common. In particular, the Grooved Pegboard and Digit Symbol tests showed improvements from preoperative to postoperative values. These measures are timed, which makes them sensitive to even subtle changes in ability levels. In addition, they are not particularly vulnerable to practice effects that could potentially distort changes in performance on re-testing. As a result, they may be more sensitive to any slowing that might be expected after microvascular changes or a disruption in blood flow during surgery. Replication of these results would be necessary before drawing conclusions about why these tests detected differences, and the direction of those differences, whereas others did not.

Both brain imaging and neurocognitive testing revealed neurologic events that one or the other may not may detect. The implication is that clinical examination is a poor method of detecting neurologic injury after high-risk cardiovascular procedures, and both brain imaging and neurocognitive testing are required for a more complete evaluation of neurologic function. The failure of brain 
imaging or neurocognitive testing to detect some lesions is not surprising given the concentration of higher cerebral functions in different areas of the brain. However, it is likely that most emboli originating from the heart and aortic valve will be evenly distributed in the brain, based on previous animal studies. ${ }^{29}$

Few studies have assessed neurocognitive decline after total arch surgery, and even fewer have examined postoperative brain imaging. ${ }^{10,14,19}$ In contrast, because of risk of embolization from calcified valves with TAVR, and awareness of transcranial Doppler data demonstrating middle cerebral artery emboli during valve insertion and deployment, ${ }^{4-6}$ postprocedure MRIs after TAVR have been extensively studied, ${ }^{1,2,7-9,30}$ although neurocognitive testing combined with brain imaging early or late has been done less frequently. These MRI studies have shown embolic events in the majority of patients, but their effect on neurocognitive decline is unclear. ${ }^{31}$ Many infarcts due to such embolic events during surgical aortic valve replacement may not be apparent on MRI, because they may be very small, and surgeons do not routinely perform MRI diffusion studies immediately postoperatively for logistic reasons.

The present study shows that posterior brain and cerebellar events may have little neurocognitive effect, but middle and anterior brain lesions are more likely to result in neurocognitive decline. Alternatively, small lesions, particularly if broadly scattered, may cause neurocognitive deficits that are not detected by brain imaging, other than perhaps evidence of increased leukomalacia and leukoaraiosis reflected as microvascular disease.

The PARTNER trial and other TAVR trials have concentrated on the subtleties of the Rankin score evaluation of patients after TAVR, including by neurologists. Our study suggests that for future trials involving procedures with a high risk of neurologic events, both postoperative brain imaging and neurocognitive studies should be performed.

\section{Study Limitations}

More events probably would have been detected if brain imaging studies or neurocognitive testing could have been done earlier postoperatively. If MRI were possible in all patients in the immediate postoperative period, it is also likely that more acute strokes would have been detected, particularly in view of the sensitivity of diffusion imaging in the first few days after the event. ${ }^{1,2,4}$ Although it is possible that many of these would be reflected as foci of gliosis on the later follow-up studies, very small acute infarcts on the diffusion study may not be visible on delayed MRI studies, such as used in this trial, and may have been of no clinical consequence.

On the basis of a previous study, we decided not to perform early postoperative neurocognitive testing because of both recovery effects and learning effects with repeated neurocognitive testing. ${ }^{14}$ Indeed, at 6 months after the procedure, many patients performed better on neurocognitive tests than they did preoperatively, likely the result of the learning effect on testing in addition to random variation. Statistical analysis of postoperative neurocognitive changes is complex and must take into account test-retest, or practice, effects. Apart from imaging and neurocognitive studies, attempts have been made to monitor brain injury with biomarkers, such as S-100 $\beta$ or multichannel EEG, but these have not shown great promise. ${ }^{14}$

We originally proposed to include delirium, seizures, and peripheral twitching among clinical neurologic events. Of note, none of these were related to findings of brain imaging or neurocognitive studies. There are reasons for these neurologic manifestations apart from brain injury, such as narcotic use, effects of sedation, and transient metabolic alterations. In a previous randomized trial, we also performed formal neurologic testing, finding that primitive reflexes could emerge transiently ${ }^{23}$; this was found to be a function of low magnesium levels occurring largely from hemodilution of cardiopulmonary bypass rather than brain injury.

\section{CONCLUSIONS}

Any study, whether clinical, brain imaging, or neurocognitive, may miss neurologic events predisposing patients to later dementia. Future studies of high-risk patients undergoing high-risk procedures should consider including all these modalities. Because of greater statistical power, this will also increase the likelihood of finding treatments that may negate the potential harmful neurologic sequelae after TAVR or aortic arch replacement.

\section{Conflict of Interest Statement}

E.E.R. receives honoraria from Terumo. J.F.S. is the North American principal investigator for the Abbott Laboratories-sponsored left main coronary disease randomized trial (EXCEL), is on the Society of Thoracic Surgeons Board of Directors, is the principal investigator for the Edwards Lifesciences (Irvine, Calif) study of the INTUITY valve (the TRANSFORM trial), receives research funding from Edwards Lifesciences, and is on the scientific advisory board of Medtronic (Minneapolis, Minn). All other authors have nothing to disclose with regard to commercial support.

\footnotetext{
References

1. Ghanem A, Muller A, Nahle CP, Kocurek J, Werner N, Hammerstingl C, et al. Risk and fate of cerebral embolism after transfemoral aortic valve implantation: a prospective pilot study with diffusion-weighted magnetic resonance imaging. J Am Coll Cardiol. 2010;55:1427-32.
} 
2. Rodes-Cabau J, Dumont E, Boone RH, Larose E, Bagur R, Gurvitch R, et al. Cerebral embolism following transcatheter aortic valve implantation: comparison of transfemoral and transapical approaches. J Am Coll Cardiol. 2011;57:18-28.

3. Knipp SC, Kahlert P, Jokisch D, Schlamann M, Wendt D, Weimar C, et al. Cognitive function after transapical aortic valve implantation: a single-centre study with 3-month follow-up. Interact Cardiovasc Thorac Surg. 2013;16: 116-22.

4. Kahlert P, Al-Rashid F, Dottger P, Mori K, Plicht B, Wendt D, et al. Cerebral embolization during transcatheter aortic valve implantation: a transcranial Doppler study. Circulation. 2012;126:1245-55.

5. Erdoes G, Basciani R, Huber C, Stortecky S, Wenaweser P, Windecker S, et al. Transcranial Doppler-detected cerebral embolic load during transcatheter aortic valve implantation. Eur J Cardiothorac Surg. 2012;41: 778-84.

6. Reinsfelt B, Westerlind A, Ioanes D, Zetterberg H, Freden-Lindqvist J, Ricksten SE. Transcranial Doppler microembolic signals and serum marker evidence of brain injury during transcatheter aortic valve implantation. Acta Anaesthesiol Scand. 2012;56:240-7.

7. Fairbairn TA, Mather AN, Bijsterveld P, Worthy G, Currie S, Goddard AJ, et al. Diffusion-weighted MRI determined cerebral embolic infarction following transcatheter aortic valve implantation: assessment of predictive risk factors and the relationship to subsequent health status. Heart. 2012; 98:18-23.

8. Astarci P, Glineur D, Kefer J, D'Hoore W, Renkin J, Vanoverschelde JL, et al. Magnetic resonance imaging evaluation of cerebral embolization during percutaneous aortic valve implantation: comparison of transfemoral and trans-apical approaches using Edwards Sapiens valve. Eur J Cardiothorac Surg. 2011;40:475-9.

9. Kahlert P, Knipp SC, Schlamann M, Thielmann M, Al-Rashid F, Weber M, et al. Silent and apparent cerebral ischemia after percutaneous transfemoral aortic valve implantation: a diffusion-weighted magnetic resonance imaging study. Circulation. 2010;121:870-8.

10. Lin R, Svensson L, Gupta R, Lytle B, Krieger D. Chronic ischemic cerebral white matter disease is a risk factor for nonfocal neurologic injury after total aortic arch replacement. J Thorac Cardiovasc Surg. 2007;133:1059-65.

11. Svensson LG, Blackstone EH, Rajeswaran J, Sabik JF III, Lytle BW, Gonzalez-Stawinski G, et al. Does the arterial cannulation site for circulatory arrest influence stroke risk? Ann Thorac Surg. 2004;78: 1274-84.

12. Svensson LG, Kim KH, Blackstone EH, Alster JM, McCarthy PM, Greenberg RK, et al. Elephant trunk procedure: newer indications and uses. Ann Thorac Surg. 2004;78:109-16.

13. Svensson LG, Nadolny EM, Kimmel WA. Multimodal protocol influence on stroke and neurocognitive deficit prevention after ascending/arch aortic operations. Ann Thorac Surg. 2002;74:2040-6.

14. Svensson LG, Nadolny EM, Penney DL, Jacobson J, Kimmel WA, Entrup $\mathrm{MH}$, et al. Prospective randomized neurocognitive and S-100 study of hypothermic circulatory arrest, retrograde brain perfusion, and antegrade brain perfusion for aortic arch operations. Ann Thorac Surg. 2001;71: 1905-12.
15. Crawford ES, Svensson LG, Coselli JS, Safi HJ, Hess KR. Surgical treatment of aneurysm and/or dissection of the ascending aorta, transverse aortic arch, and ascending aorta and transverse aortic arch. Factors influencing survival in 717 patients. J Thorac Cardiovasc Surg. 1989;98:659-74.

16. Smith CR, Leon MB, Mack MJ, Miller DC, Moses JW, Svensson LG, et al Transcatheter versus surgical aortic-valve replacement in high-risk patients. N Engl J Med. 2011;364:2187-98.

17. Leon MB, Smith CR, Mack M, Miller DC, Moses JW, Svensson LG, et al Transcatheter aortic-valve implantation for aortic stenosis in patients who cannot undergo surgery. N Engl J Med. 2010;363:1597-607.

18. Svensson LG, Crawford ES, Hess KR, Coselli JS, Raskin S, Shenaq SA, et al Deep hypothermia with circulatory arrest. Determinants of stroke and early mortality in 656 patients. $J$ Thorac Cardiovasc Surg. 1993;106:19-31.

19. Uysal S, Mazzeffi M, Lin HM, Fischer GW, Griepp RB, Adams DH, et al Internet-based assessment of postoperative neurocognitive function in cardiac and thoracic aortic surgery patients. J Thorac Cardiovasc Surg. 2011;141: 777-81.

20. Haacke EM, Xu Y, Cheng YC, Reichenbach JR. Susceptibility weighted imaging (SWI). Magn Reson Med. 2004;52:612-8.

21. Wahlund LO, Barkhof F, Fazekas F, Bronge L, Augustin M, Sjogren M, et al. A new rating scale for age-related white matter changes applicable to MRI and CT. Stroke. 2001;32:1318-22.

22. Wilkinson GS, Robertson GJ. WRAT4 Professional Manual. Lutz, FL: Psychological Assessment Resources; 2006.

23. Bhudia SK, Cosgrove DM, Naugle RI, Rajeswaran J, Lam BK, Walton E, et al. Magnesium as a neuroprotectant in cardiac surgery: a randomized clinical trial J Thorac Cardiovasc Surg. 2006;131:853-61.

24. Heaton RK, Grant I, Matthews CG. Comprehensive Norms for an Expanded Halstead-Reitan Battery: Demographic Corrections, Research Findings, and Clinical Applications. Odessa, FL: Psychological Assessment Resources; 1991.

25. Kneebone AC, Andrew MJ, Baker RA, Knight JL. Neuropsychologic changes after coronary artery bypass grafting: use of reliable change indices. Ann Thorac Surg. 1998;65:1320-5.

26. Rubin DB. Multiple Imputation for Non-response in Surveys. New York: Wiley; 1987.

27. Breiman L. Bagging predictors. Machine Learning. 1996;24:123-40.

28. Sauerbrei W, Schumacher M. A bootstrap resampling procedure for model building: application to the Cox regression model. Stat Med. 1992;11: 2093-109.

29. Svensson LG, Robinson MF, Esser J, Fritz VU, Levien LJ. Influence of anatomic origin on intracranial distribution of micro-emboli in the baboon. Stroke. 1986; 17:1198-202.

30. Meller SM, Baumbach A, Voros S, Mullen M, Lansky AJ. Challenges in cardiac device innovation: is neuroimaging an appropriate endpoint? Consensus from the 2013 Yale-UCL Cardiac Device Innovation Summit [abstract]. BMC Med. 2013; $11: 257$.

31. Purandare N. Preventing dementia: role of vascular risk factors and cerebral emboli. Br Med Bull. 2009;91:49-59.

Key Words: brain protection, circulatory arrest, aortic arch 


\section{APPENDIX E1. PROTOCOL \\ Prospective Randomized Study of Brain Protection During Aortic Arch Replacement}

Principal Investigator

Dr Lars Svensson, MD, PhD

Staff Cardiac Surgeon

Department of Thoracic and Cardiovascular Surgery

Biostatistician

Carolyn Apperson-Hansen, MStat

Department of Biostatistics and Epidemiology

Amendment 2, July 15, 2009

Contents

1. Introduction 1147.e1

2. Background 1147.e1

3. Hypothermic circulatory arrest 1147.e1

4. Antegrade brain perfusion 1147.e1

5. Retrograde brain perfusion $1147 . \mathrm{e} 2$

6. Methods $1147 . \mathrm{e} 2$

7. Statistical methods 1147.e4

8. References $1147 . e 4$

9. Appendix $1147 . e 5$

\section{INTRODUCTION}

The purpose of the study is to assess the best method of protecting the brain during long periods of circulatory arrest. The aim is to determine which method of brain perfusion (1) retrograde brain perfusion (RBP) or (2) antegrade brain perfusion (ABP) via the right subclavian artery, during hypothermic circulatory arrest (HCA) is better at protecting the brain against injury after total aortic arch replacement. The outcome will be determined by the composite incidence of stroke, neuropsychological deficits (termed as neurobehavioural outcome), and mortality. The battery of tests for the neuropsychological assessment is detailed in the Methods section.

The proposed study is a randomized study comparing RBP and ABP during HCA in patients undergoing total aortic arch replacement. Patient follow-up will be at discharge and 6 months postprocedure. The primary endpoint for the study is freedom from postoperative neurobehavioural deficit and mortality at 6 months.

\section{BACKGROUND}

The challenge in aortic arch repair is the requirement to preserve cerebral function despite the need to manipulate the cerebral circulation. It is important to not only prevent global ischemic injury but to also prevent dislodgement of thrombus and atherosclerotic debris into the cerebral circulation.

Aortic arch repair may require interruption of the cerebral circulation not only during aortic anastomoses but also during dissection and mobilization of the aortic arch and cerebral vessels.

Various strategies have evolved for protecting the brain during the interval in which its normal circulation is interrupted. These strategies all involve hypothermia and can be categorized into:

1. Complete interruption of flow or hypothermic circulatory arrest (HCA) alone,

2. Addition of selective antegrade brain perfusion (ABP),

3. Addition of retrograde brain perfusion (RBP).

\section{HYPOTHERMIC CIRCULATORY ARREST}

Hypothermic circulatory arrest (HCA) was introduced as an alternative to extracorporeal circulation. ${ }^{\mathrm{E} 1 \mathrm{E} 2}$ The efficacy of HCA has been widely accepted in protecting the brain in adults undergoing aortic aneurysm repair and in children undergoing congenital heart lesions repair. ${ }^{\mathrm{E}, \mathrm{E} 4}$ With increased use of HCA some of the limitations were revealed, mainly its safety during longer durations of arrest. This has led to interest in investigation of HCA and in exploration of alternative techniques such as antegrade brain perfusion (ABP) and retrograde brain perfusion (RBP).

Permanent injury to the brain may occur if brain circulation is arrested for longer than 60 minutes at $18^{\circ} \mathrm{C}$. ${ }^{\mathrm{E} 5}$

Results show that patients who have surgical correction of congenital heart defects using HCA generally emerge from the operation without serious neurologic sequelae; however, careful studies using standardized tests of behavior and learning in young children show that some loss of cognitive function may occur especially with prolonged periods of circulatory arrest. ${ }^{\mathrm{E} 6, \mathrm{E} 7}$

The consensus is that a period of circulatory arrest longer than 40 or 50 minutes raises concerns over brain protection. Circulatory arrest for longer than 60 minutes is universally regarded as risky. ${ }^{\text {E12 }}$ It is generally accepted that too short a period of cooling may result in damage due to uneven and inadequate lowering of brain temperature. Packing the head with ice during prolonged periods of circulatory arrest improves outcome.

Hypothermia not only protects the brain by suppressing the metabolic rate but also by suppression of release of cytotoxic amino acids or free oxygen radicals. ${ }^{\text {E14 }}$

Following HCA there is inappropriate vasoconstriction, which is believed to be involved in producing brain injury. To avoid this vasoconstriction an initial period of cold perfusion is instituted. ${ }^{\mathrm{E} 15}$

\section{Monitoring}

Cerebral electrical function is monitored by means of an electroencephalogram (EEG) or computer derived monitoring. A silent EEG determines adequate cooling; this is done before institution of circulatory arrest.

\section{ANTEGRADE BRAIN PERFUSION (ABP)}

Historically, brain perfusion was achieved by using several pumps and cannulating both subclavian arteries and both carotid arteries. ${ }^{\text {E16 }}$ This technique was abandoned due to problems associated with controlling pressure and flow in these 
separate vascular beds to ensure relatively uniform perfusion, with resulting poor outcome for the patients.

There is renewed interest in ABP as HCA is not safe for long durations. It is recognized that combining $\mathrm{ABP}$ and hypothermia allows use of much lower flow rates and that low-flow hypothermic antegrade perfusion may provide better brain protection from global ischemia than HCA. ${ }^{\mathrm{E} 8-\mathrm{E} 11, \mathrm{E} 13, \mathrm{E} 17, \mathrm{E} 18}$

Mortality after using ABP ranges from 5\%-19\%, stroke $1 \%-4 \%$, and transient neurologic lesions $4 \%-6 \% .^{\text {E19-E22 }}$

\section{RETROGRADE BRAIN PERFUSION (RBP)}

Retrograde brain perfusion has the potential of reducing embolization to the brain. ${ }^{\text {E23,E24 }}$ Theoretically, its implementation can allow longer durations of HCA. Research has shown that very little of the perfusate actually reaches the brain. Most of the retrograde flow is shunted from the superior vena cava to inferior vena cava via the azygous system. ${ }^{\text {E25 }}$ Some retrograde perfusion does occur via the collaterals surrounding the spinal cord and reaching the brain via the vertebral veins. Less than $5 \%$ of retrograde flow returns via the arch vessels, probably too inadequate to meet the ongoing metabolic demands of the brain, even in the presence of hypothermia. ${ }^{\mathrm{E} 10}$ It seems unlikely that the rate of RBP can be manipulated clinically high enough to provide complete nutritive maintenance during arrest of antegrade flow. However, RBP still has clinical appeal as a possible measure to prevent or ameliorate injury. Furthermore, it does appear to be effective in flushing out embolic material from the aortic arch greater vessels.

It is clear that some perfusion of the brain, antegrade or retrograde, during hypothermic circulatory arrest is associated with improved outcome compared to HCA alone.

Improvements in techniques have led to a reduction in the risk of stroke, although, in a study of 656 patients undergoing deep hypothermia with circulatory arrest, it was noted that $7 \%$ suffered a stroke after the operation. ${ }^{\text {E26 }}$ The incidence of neurocognitive deficits in these patients has not been clearly documented. In a prospective randomized study, carried out by the investigators, of patients undergoing short periods of circulatory arrest there was no difference in neuropsychologic function when HCA, RBP, and ABP were compared. ${ }^{\text {E27 }}$ We believe it would be important to compare $\mathrm{RBP}$ and $\mathrm{ABP}$ for prolonged periods of circulatory arrest during replacement of the entire aortic arch.

Currently, the decision to use HCA with RBP or ABP is made by the surgeon. There is no national standard of practice related to choosing one method over the other. At The Cleveland Clinic Foundation (CCF) the perfusion team is skilled at HCA with both RBP and ABP.

\section{METHODS}

\section{Study Design}

This is a prospective randomized study of patients who require deep hypothermic circulatory arrest for total aortic arch replacement. Patients will be randomized to receive either retrograde brain perfusion or antegrade perfusion by the right subclavian artery. All the patients will be operated on at the Cleveland Clinic Foundation.

\section{Study Objective}

The primary endpoint is decline in neurobehavioral outcome, based on the presence of cerebrovascular accident (CVA) immediate postsurgery, inhospital death from neurological causes and/or neuropsychological assessment at 180 days. Cerebrovascular accident and/or death from neurological causes postsurgery included only if it occurs during the index hospitalization, while the patient is recovering from the study procedure. A patient is considered to have declined neurobehaviorally if he/she has died from neurological causes or has had a cerebrovascular accident or has two or more reliable change (RC) indices that fall below the lower $90 \%$ confidence limit for the respective, corrected RC interval. The calculation for obtaining RC indices is outlined in Appendix A.

\section{Inclusion/Exclusion Criteria}

Patients will be eligible for enrollment into the study if they meet the following inclusion and exclusion criteria.

\section{i. Inclusion criteria:}

Patients must meet all of the following requirements to be eligible for enrollment in the study:

- Undergoing a total aortic arch replacement.

- Patient is less than 75 years old.

\section{ii. Exclusion criteria:}

Patients will be excluded from the study if:

- Cardiac ejection fraction is less than $35 \%$

- Patient shows evidence of major illness such as severe hepatic disease, severe renal failure, active cancer, or active major infection

- Patient unable to complete preoperative assessment

- Patient is not willing or able to complete all follow-up requirement

- Patient is already enrolled in other new device or drug investigational protocols that have not completed the primary endpoint or that clinically interferes with study endpoints.

- Patient is a female who is pregnant or lactating

- Patient has a history of stroke

An informed consent will be obtained from the patients.

\section{Study Scope and Duration}

This is a single center randomized study with enrollment targeted to be completed within 2 years, and final follow-ups complete within 8 months after the last patient is randomized.

\section{Risk/Benefit Analysis}

All patients will be undergoing extensive aortic surgery and the risks entailed would be those of deep hypothermia and circulatory arrest with risk of death of approximately $10 \%$ and risk of stroke of approximately $7 \%$. It has been found that keeping retrograde brain perfusion rates at $500 \mathrm{cc} / \mathrm{min}$ and the central venous pressure below $25-30 \mathrm{~cm}$ of water reduces complications such as brain edema. The risk with cannulation of the right subclavian artery would be the technical ones involved with cannulating the artery and repairing it. Risk of injuring the brachial-plexus is most unlikely and rare since the brachial-plexus is some distance away from this area. We currently use both ABP and RBP with no serious technical complications.

\section{Study Procedure}

\section{i. Initial patient screening}

Subjects will be evaluated for eligibility by the Investigator prior to entry into the Study per applicable Inclusion and Exclusion Criteria. 
All the patients undergoing total arch repair routinely undergo brain magnetic resonance imaging (MRI) studies and cardiac echocardiograms prior to the surgery.

\section{ii. Informed consent}

If the subject is determined to be a potential candidate, the Clinical Investigator or Research Team Personnel shall approach the patient and review and explain the proposed procedure and Patient Informed Consent. The potential benefits and risks of aortic arch surgery will be carefully explained to the patient and any questions answered. If agreed, the subject will then be asked to sign the Patient Informed Consent.

\section{Study Procedures}

For those patients who meet the initial eligibility criteria and agree to participate by signing the Patient Informed Consent, the following will be performed:

\section{i. Baseline evaluation}

The Investigators will conduct a Baseline Evaluation of each candidate, which will include a battery of neuropsychological testing. A certified person will perform the neuropsychological examination. The neuropsychological assessment will include the following battery of tests:
a. Depression Inventory Test
b. Hopkins Verbal Learning
c. Grooved Pegboard Test (Peg R, Peg L, Peg RL)
d. Controlled Oral Word Association Test
e. Trail Making Test (TMT A and TMT B)
f. Kaufman Brief Intelligence Test
g. Digit Symbol Subtest and Symbol Search Test
h. Boston Naming Test
i. National Adult Reading Test-Revised (only at baseline)

Patients will be contacted by telephone at 1 month to determine the occurrence of adverse events since discharged.

The second assessment will be at 6 months postsurgery. For patients requiring a second stage surgery, neurocognitive assessment will be performed at 90 days $( \pm 30$ days $)$ from first surgery. The preoperative and 6 month postsurgery battery will include the 8 tests mentioned above. National Adult Reading Test-Revised will only be done at baseline. It takes approximately 45 minutes to administer all tests listed above.

Presence of CVA will be assessed by a neurologist (during the index hospitalization) and confirmed with the appropriate imaging technique. This is part of routine, post-operative standard of practice in patients having cardiac or aortic surgery at CCF.

\section{ii. Surgical procedure}

Immediately before the operation each patient will be randomized to either the retrograde or the antegrade brain perfusion groups. Once randomized, the patient will undergo surgery using the standard methods and procedures for that study arm. The circulation will then be arrested and the aorta opened and the operative procedure performed. In patients with retrograde brain perfusion, this involves switching arterial perfusion from the femoral artery to the superior vena cava, which is done by moving clamps from one circuit to the other circuit. This is standard practice that has been used for a number of years. In patients that have been randomized to antegrade brain perfusion, the standard method of perfusion through the right subclavian artery will be performed and a balloon catheter will be placed in the origin of the innominate artery to prevent the blood from flowing into the operative field. The rest of the operation will be conducted as required using standard methods.

The only difference between this study and our current standard of care is that patients will be randomized to one of two methods of brain perfusion and have neuropsychological testing before surgery and at 90 days or 6 months after surgery, depending on type of surgery.

\section{iii. Postprocedure follow-up and evaluation}

Patients will be managed as per Cleveland Clinic Foundation Thoracic and Cardiovascular Surgery Department protocols postoperatively.
All cardiac, neurological, renal, and gastrointestinal complications are documented in the patient's medical record and treated as per the departmental protocols. This is our current standard of practice at CCF; this study will not require an alteration in assessment or treatment of inpatient complications.

Patients are also contacted at 1 month by telephone.

At $180( \pm 30)$ days postprocedure the patient will return for neuropsychological tests. Patients requiring second stage surgery will return at 90 $( \pm 30)$ for neuropsychological tests.

The proposed study includes the following interactions with patients, interventions and assessments:

a. Days presurgery (3 or 4): Consent followed by neuropsychological assessment

b. Day of surgery: Randomization to treatment or control

c. Postsurgery while still inpatient: Routine care as per CCF protocol.

d. Routine data collection, as per Cleveland Clinic Foundation Thoracic and Cardiovascular Surgery Department protocol will be collected for presurgery, surgery, and postsurgery (inpatient) periods. Registry and databases for this are Internal Review Board approved (\# 4826).

e. 90 or $180 \pm 30$ days postsurgery: Neuropsychological assessment

On discharge from hospital patients will be instructed about the follow-up neuropsychological assessment at 90 or 180 days from the time of surgery.

\section{Adverse Events}

Adverse events (AE) will be classified into serious and nonserious events on strictly objective definitions. Adverse events will be recorded in our departmental databases per Departmental Registries policies, procedures and Standard of Practices.

\section{Adverse Events Definitions}

\section{i. Adverse event}

An AE is any undesirable medical event occurring to a patient in a clinical study, whether or not considered related to the study procedure. This includes events not seen at baseline or worsened if present at baseline.

\section{ii. Serious adverse event (SAE)}

A SAE is any adverse experience that results in any of the following outcomes:

a. Death. This is any death occurring from the time of surgery to the end of the follow-up period.

b. A life-threatening experience. This refers to an event in which the patient was at risk of death at the time of the event. However, does not refer to an event that hypothetically might have caused death if it was more severe.

c. A persistent or significant disability/incapacity

d. Requires prolongation of existing hospitalization

\section{iii. Nonserious adverse events}

A nonserious AE is any adverse event that does not fulfill the definition of an SAE.

\section{iv. Severity assessment definitions}

a. Mild-Transient symptoms, no interference with the patient's daily activities.

b. Moderate-Marked symptoms, moderate interference with the patient's daily activities.

c. Severe-Considerable interference with the patient's daily activities.

Adverse Events that could potentially occur during this study are listed below:

a. Death. This is any death occurring from the time of surgery to the end of the follow-up period.

b. Stroke or any neurological event. 
c. Injury to right subclavian artery.

d. Adverse events related to the use of cardiopulmonary bypass.

\section{v. Measures taken by the investigator to reduce the po-} tential risks to the patient

Selecting eligibility criteria that will exclude those patients at highest risk of experiencing an anticipated adverse event.

Patients will be monitored closely postoperatively, including assessment of neurologic status.

A neurologist will see any patient with clinical postoperative neurologic deficit.

\section{Data Collection}

Routinely, data are collected pre-, intra-, and postoperatively by Registries personnel and entered into departmental databases. In addition, data will be collected and entered into a separate database, designed for this study, to capture nondepartmental database information (neuropsychological testing results).

The Department of Biostatistics will collaborate with the Department of Thoracic and Cardiovascular Surgery in development of data collection instruments as well as the database.

\section{i. Components of the database}

a. Neuropsychological assessment variables (preoperative and 3 or 6 months postsurgery)

All other variables will be obtained from departmental databases, including adverse events and mortality information. Special database created for this study will not use patient name, only CCF patient ID number. In this way, data can be linked up to data from the departmental registry/databases for analysis. All data collected in the special database will be housed on the department server, which is password protected.

\section{STATISTICAL METHODS}

\section{i. Randomization}

Patients will be randomized 1:1 to the two patient groups.

Recruitment will take place at the time of the preoperative visit (ie, 3-4 days prior to surgery). Randomization will be done in the operation room. A patient will be considered to be randomized at induction of anaesthesia.

\section{ii. Sample Size Justification}

The potential patient population for this single center study is very small as is demonstrated by the numbers of patients who would have been eligible for this trial in the past few years ( 14 in 1999, 24 in 2000, 31 in 2001, and 54 by October 2002). Further, it is expected that the composite incidence of stroke, neuropsychological deficits (termed as neurobehavioural outcome), and mortality will be $45 \%$ in the RBP group and $16 \%$ in the ABP group. This translates into a relative risk of 2.8. Assuming a relative risk of $2.5,16 \%$ incidence in the ABP group, power of $80 \%$, a significance level of 0.05 , and two-sided testing, the sample size needed is 108, 54 randomized to each group. Assuming $20 \%$ loss to follow-up, the total sample size is 130,65 per group.

\section{iii. Analysis}

Simple descriptive statistics will be used to summarize the data. For categorical variables, this will include frequencies and percentages. For continuous variables, this will include the mean, median, and standard deviation.
The primary endpoint is the occurrence of neurological decline, based on the neurological presence of CVA immediately postsurgery, neuropsychological assessment at 90 or 180 days postsurgery, and/or death from neurological causes during the immediate postop period. A patient will be considered to have declined neurologically if he/she has died from neurological causes or has two or more reliable change (RC) indexes that fall below the lower $90 \%$ confidence limit for the respective, corrected RC interval. ${ }^{\mathrm{E} 28}$ Appendix A outlines the method for RC index calculation.

To test neurological decline and/or death from neurological causes between the two groups, a statistical test for two independent proportions will be conducted. Initially, unadjusted logistic regression for any perceived baseline characteristics and/or prespecified covariates will be used. The odd ratio (with 95\% confidence interval) will be reported. Secondary analysis of the primary endpoint will be a multivariable logistic regression.

A secondary endpoint analysis of time to CVA or death from surgery will be performed.

Baseline characteristics will be assessed for balance between the two study groups. Any characteristic deemed to be clinically significant will become a potential covariate.

Exploratory data analyses will be conducted on areas deemed clinically appropriate. Statistical methods could include chi-square, logistic regression, and ANOVA methods.

Unless otherwise stated, analyses will be conducted in the intent to treat population and statistical testing will have two-sided alternatives with a type I error level of 0.05 .

Statistical analysis will be conducted using the SAS package.

\section{E-References}

E1. Borst HG, Schaudig A, Rudolph W. Arteriovenous fistula of the aortic arch: repair during deep hypothermia and circulatory arrest. J Thorac Cardiovasc Surg. 1964;3:443.

E2. Lillehei CW, Todd DB, Levy MJ, Ellis RJ. Partial cardiopulmonary bypass, hypothermia and total circulatory arrest: a lifesaving technique for ruptured mycotic aneurysms, ruptured left ventricle, and other complicated aortic pathology. J Thorac Cardiovasc Surg. 1969;58:530.

E3. Barrat-Boyes BG, Simpson M, Neutze JM. Intracardiac surgery in neonates and infants using deep hypothermia with surface cooling and limited cardiopulmonary bypass. Circulation. 1971;43(suppl):I25-30.

E4. Griepp RB, Stinson EB, Hollingsworth JF, Buehler D. Prosthetic replacement of the aortic arch. J Thorac Cardiovasc Surg. 1975;70:1051.

E5. Michenfelder JD, Milde JH. The relationship among canine brain temperature, metabolism, and function during hypothermia. Anesthesiology. 1991;75:130.

E6. Wells FC, Coghill S, Caplan HL, Lincoln C. Duration of circulatory arrest does influence the psychological development of children after cardiac operation in early life. J Thorac Cardiovasc Surg. 1983;86:823.

E7. Bellinger DC, Jonas AR, Rapport LA, et al. Developmental and neurological status of children after heart surgery with hypothermic circulatory arrest or low-flow cardiopulmonary bypass. N Engl J Med. 1995;332:549.

E8. Mault JR, Ohtake S, Klingensmith MF, et al. Cerebral metabolism and circulatory arrest: effects of duration and strategies for protection. Ann Thorac Surg. 1993;55:57.

E9. Mezrow CK, Midulla PS, Sadeghi AM, et al. Quantitative electroencephalog raphy: a method to assess cerebral injury after hypothermic circulatory arrest. J Thorac Cardiovasc Surg. 1995;109:925. 
E10. Midulla PS, Gandsas A, Sadeghi AM, et al. Comparison of retrograde cerebral perfusion to antegrade cerebral perfusion and hypothermic circulatory arrest in a chronic porcine model. J Card Surg. 1994;9:560.

E11. Mezrow CK, Gandsas A, Sadeghi AM, et al. Metabolic correlates of neurologic and behavioral injury after prolonged hypothermic circulatory arrest. J Thorac Cardiovasc Surg. 1995;109:959.

E12. Ergin MA, Griepp EB, Lansman SL, et al. Hypothermic circulatory arrest and other methods of cerebral protection during operations on the thoracic aorta. $J$ Card Surg. 1994;9:525.

E13. Kawata H, Fackler JC, Aoki M, et al. Recovery of cerebral blood flow and energy state in piglets after hypothermic circulatory arrest versus recovery after low flow bypass. J Thorac Cardiovasc Surg. 1993;106:671.

E14. Griepp EB, Griepp RB. Cerebral consequences of hypothermic circulatory arrest in adults. J Card Surg. 1992;7:134.

E15. Jonassen AE, Quaegebeur JM, Young WL, et al. Cerebral blood flow velocity in pediatric patients is reduced after cardiopulmonary bypass with profound hypothermia. J Thorac Cardiovasc Surg. 1995;110:934.

E16. DeBakey ME, Crawford ES, Cooley DA, Morris GC. Successful resection of fusiform aneurysm of aortic arch with replacement of homograft. Surg Gynecol Obstet. 1957;105:657.

E17. Filgueiras CL, Winsborrow B, Ye J, et al. A 31 P magnetic resonance study of antegrade and retrograde cerebral perfusion during aortic arch surgery in pigs. $J$ Thorac Cardiovasc Surg. 1995;110:55.

E18. Swain JA, McDonald TJ, Griffith PK, et al. Low-flow hypothermic cardiopulmonary bypass protects the brain. J Thorac Cardiovasc Surg. 1991;102:76.

E19. Bachet J, Guilmet D, Goudot B, et al. Cold cerebroplegia: a new technique of cerebral protection during operations on the transverse aortic arch. J Thorac Cardiovasc Surg. 1991;102:85.

E20. Matsuda H, Nakano S, Shiraku R, et al. Surgery for aortic arch aneurysm with selective cerebral perfusion and hypothermic cardiopulmonary bypass. Circulation. 1989;80(suppl I):I243-8

E21. Kazui T, Kimura N, Yamada O, Komatsu S. Surgical outcome of aortic arch aneurysms using selective cerebral perfusion. Ann Thorac Surg. 1994;57:904.

E22. Tabayashi K, Ohmi M, Togo T, et al. Aortic arch aneurysm repair using selective cerebral perfusion. Ann Thorac Surg. 1994;57:1305.

E23. Lemole GM, Strong MD, Spagna PM, et al. Improved results for dissecting aneurysms: intraluminal sutureless prosthesis. J Thorac Cardiovasc Surg. 1982:83:249.

E24. Mills NL, Ochsner JL. Massive air embolism during cardiopulmonary bypass: causes, prevention and management. J Thorac Cardiovasc Surg. 1980;80:708.

E25. de Brux JL, Subayi JB, Pegis JD, Pillet J. Retrograde cerebral perfusion: anatomic study of the distribution of blood to the brain. Ann Thorac Surg. 1995;60:1294.

E26. Svensson LG, Crawford ES, Hess KR, et al. Deep hypothermia with circulatory arrest: determinants of stroke and early mortality in 656 patients. J Thorac Cardiovasc Surg. 1993;106:19.

E27. Svensson LG, Nadolny EM, Penney DL, et al. Prospective randomized neurocognitive and S-100 study of hypothermic circulatory arrest, retrograde brain perfusion, and antegrade brain perfusion for aortic arch operations. Ann Thorac Surg. 2001;71:1905.

E28. Kneebone AC, Andrew MJ, Baker RA, Knight JL. Neuropsychologic changes after coronary artery bypass grafting: use of reliable change indices [see comments]. Ann Thorac Surg. 1998;65:1320-5.

\section{APPENDIX A. (OF PROTOCOL) Calculation of Reliable Change Indices}

Neurological and neuropsychological assessment results will be compared between the preoperative and the postoperative results. ${ }^{\mathrm{E} 28}$ Analysis of the data will involve calculating the reliable change indices (RC). The $\mathrm{RC}$ index will be calculated for each test using the baseline and follow-up data. The test-retest reliability coefficient $\left(\mathrm{r}_{\mathrm{xx}}\right)$ will be computed for each measure (Pearson correlation between preoperative and postoperative scores), from which the standard error of measurement $\left(\mathrm{SE}_{\mathrm{m}}\right)$ will be calculated using the formula
$\mathrm{SE}_{\mathrm{m}}=\mathrm{SD}_{1}\left(\sqrt{ }\left[1-\mathrm{r}_{\mathrm{xx}}\right]\right)$, where $\mathrm{SD}_{1}$ is the standard deviation (SD) of the baseline score. The standard error (SE) of the difference $\left(\mathrm{SE}_{\mathrm{diff}}\right)$ will then be obtained using $\mathrm{SE}_{\text {diff }}=\sqrt{ }\left[2\left(\mathrm{SE}_{\mathrm{m}}\right)^{2}\right]$.

Standard error of the difference describes the spread of the distribution of change scores that would be expected if no actual change had occurred. The $\mathrm{SE}_{\text {diff }}$ will be multiplied by \pm 1.64 SD to establish a $90 \%$ RC confidence interval (2-tailed prediction), in which only $5 \%$ of cases would be expected to be above and $5 \%$ to be below the cutoff. A correction representing the practice effect will then be added to the 2-tailed cutoff points. The practice effect will be calculated for each variable as the mean difference between the follow-up and baseline scores. The following formula will then be used to calculate the RC $90 \%$ confidence interval for each variable:

$$
\mathrm{RC} \text { interval }=\left(\mathrm{SE}_{\text {diff }}\right) *( \pm 1.64)+\text { practice effect }
$$

Resulting cutoff values will be rounded to the nearest whole number outside the $90 \%$ RC interval. For each patient, a postoperative minus preoperative difference score will be calculated for each neuropsychological measure. If this score falls outside the RC interval, a statistically significant change in performance on that measure will be considered to have occurred.

APPENDIX E2. Neuropsychologic measures and cognitive domains assessed

\begin{tabular}{|c|c|}
\hline Neuropsychologic measure & Cognitive domain assessed \\
\hline $\begin{array}{l}\text { Hopkins Verbal Learning } \\
\text { Test-revised* }\end{array}$ & $\begin{array}{l}\text { Attention, learning, } \\
\text { and memory }\end{array}$ \\
\hline $\begin{array}{l}\text { Grooved Pegboard Test } \\
\qquad(\text { Peg R, Peg L, Peg RL }) \dagger\end{array}$ & Timed manual dexterity \\
\hline $\begin{array}{c}\text { Controlled Oral Word } \\
\text { Association Test } t+\end{array}$ & Timed word fluency \\
\hline Trail Making Test (Form A) $\S$ & Visuomotor speed, sequencing \\
\hline Trail Making Test (Form B) $\S$ & $\begin{array}{l}\text { Visuomotor speed, sequencing, } \\
\text { and set-shifting }\end{array}$ \\
\hline $\begin{array}{l}\text { Digit Symbol Subtest and } \\
\text { Symbol Search Subtest } \mid\end{array}$ & Timed visuomotor coordination \\
\hline Boston Naming Test $\Upsilon$ & Confrontation naming \\
\hline Beck Depression Inventory-II\# & Depression \\
\hline $\begin{array}{l}\text { Wide Range Achievement } \\
\text { Test (4th ed)*** }\end{array}$ & Single word reading \\
\hline \multicolumn{2}{|c|}{$\begin{array}{l}\text { *Brandt J, Benedict RB. Hopkins Verbal Learning Test-Revised. Administration } \\
\text { Manual. Lutz, FL: Psychological Assessment Resources; 2001. †Klove H. Clinical } \\
\text { neuropsychology. In: Forster FM, ed. The Medical Clinics of North America. New } \\
\text { York: Saunders; 1963. łBenton AL, Hamsher K. Multilingual Aphasia Examination } \\
\text { Manual. Iowa City: University of Iowa; 1978. §Army Individual Test Battery. Manual } \\
\text { of Directions and Scoring. Washington, DC: War Department, Adjutant General’s } \\
\text { Office; 1944. ||Wechsler D. Wechsler Adult Intelligence Scale. 3rd ed. San Antonio, } \\
\text { TX: The Psychological Corporation; 1997. } \mid \text { Goodglass H, Kaplan E, Barresi B. Bos- } \\
\text { ton Diagnostic Aphasia Examination Manual. 3rd ed. San Antonio, TX: Pearson; } \\
\text { 2000. \#Beck AT, Steer RA, Brown GK. Manual for the Beck Depression Inven- } \\
\text { tory-II. San Antonio, TX: Psychological Corporation; 1996. **Wilkinson GS, Rob- } \\
\text { ertson GJ. WRAT4 Professional Manual. Lutz, FL: Psychological Assessment } \\
\text { Resources; 2006. }\end{array}$} \\
\hline
\end{tabular}




\section{APPENDIX E3. MULTIPLE IMPUTATION FOR COMPONENTS OF COMPOSITE END POINT AND SENSITIVITY ANALYSIS}

Because not every patient had brain imaging and neurologic studies, data for brain imaging change and some components of neurocognitive decline were missing for 26 and 25 patients, respectively (Table 2). This resulted in 31 cases with missing data in the composite end point. Therefore, we adopted the following multiple imputation strategy, and sensitivity analysis, to impute the missing in-hospital events.

\section{METHODS}

Using patient, procedure, and components of the composite end point (Appendix E4) and a 10-fold multiple imputation model ${ }^{26}$ (SAS PROC MI, SAS Institute Inc, Cary, NC), we imputed missing data for brain imaging change and components of neurocognitive decline. Imputation was performed separately for each perfusion group using the Markov Chain Monte Carlo method under the assumption that missing values are missing at random. Imputed events were then dichotomized using a cutoff criterion of 0.5 , and the imputed components and resulting composite end point were tested for significance using a logistic regression model (SAS PROC LOGISTIC) for each imputed data set. Finally, following Rubin, ${ }^{26}$ we combined the regression estimates from the 10 models (SAS PROC MIANALYZE) to obtain the final estimates and the $P$ value.

In a sensitivity analysis, we repeated the whole analysis process under liberal $(0.25)$ and conservative $(0.75)$ cutoff criteria.

\section{RESULTS}

Table E9 shows the range of frequency of imputed components over the 10 imputed data sets. The number of patients with brain imaging changes ranged from 18 to 21 , and the number of patients with neurocognitive decline ranged from 19 to 20 . The resulting frequency of composite event ranged from 36 to 38 .

Comparison of brain imaging changes between $\mathrm{ABP}$ and RBP using the 10-fold imputed data showed no statistically significant difference in frequencies between the methods $(P=.16)$. Likewise, no significant difference $(P=.6)$ was observed between the brain protection methods in neurocognitive decline. Finally, no significant difference was observed in the composite end point $(P=.2$, ranging from .10 to .3 over the 10 imputed data sets).

In the sensitivity analysis, liberal and conservative cutoff criteria yielded results similar to those of the mid-point analysis.

\section{APPENDIX E4. VARIABLES CONSIDERED IN MULTIPLE IMPUTATION MODEL \\ Demography}

Age (y), sex, race, body mass index $\left(\mathrm{kg} \cdot \mathrm{m}^{-2}\right)$.

\author{
Acuity \\ New York Heart Association functional class (I-IV), \\ emergency surgery.
}

\section{Cardiac Comorbidity}

Prior myocardial infarction, atrial fibrillation/flutter, complete heart block or pacer, heart failure.

\section{Noncardiac Comorbidity}

Peripheral arterial disease, carotid disease, hypertension, chronic obstructive pulmonary disease, history of smoking, prior stroke, bilirubin $\left(\mathrm{mg} \cdot \mathrm{dL}^{-1}\right)$, cholesterol $\left(\mathrm{mg} \cdot \mathrm{dL}^{-1}\right)$, high-density lipoprotein cholesterol $\left(\mathrm{mg} \cdot \mathrm{dL}^{-1}\right)$, triglycerides $\left(\mathrm{mg} \cdot \mathrm{dL}^{-1}\right)$, creatinine $\left(\mathrm{mg} \cdot \mathrm{dL}^{-1}\right)$, blood urea nitrogen $\left(\mathrm{mg} \cdot \mathrm{dL}^{-1}\right)$, hematocrit $(\%)$.

\section{Perfusion}

Percent time ABP, temperature (nasopharyngeal low, bladder low, bladder high, blood [Swan-Ganz] high, venous blood low, venous blood high), cooling time (minutes), warming time (minutes).

\section{Support (Interval)}

Total myocardial ischemic time (minutes), total cardiopulmonary bypass time (minutes), total circulatory arrest time (minutes)

\section{Magnetic Resonance Imaging Score (Preoperative, Postoperative)}

Anterior brain, middle brain, posterior brain $(0=$ none, $1=$ mild, $2=$ moderate, $3=$ severe).

\section{Neurocognitive Test (Preoperative, Postoperative)}

Hopkins Verbal Learning Test (HVLT) trial 1 (raw score, $z$ value), HVLT total (raw score, $z$-value), HVLT delayed recall (raw score, $z$-value), HVLT discrimination index (raw score, $z$-value), Controlled Oral Word Association Test-FAS (raw score, adjustment, raw score with age-education bonus, $z$ value), Boston Naming Test (total score, total score with cues, $z$-value), Pegs Dominant Hand Test (raw score [in seconds], scaled score, $z$-value), Pegs Nondominant Hand Test (raw score [in seconds], scaled score, $z$-value), Digit Symbol Coding (raw score, scaled score, $z$-value), Symbol Search (raw score, scaled score, $z$-value), Processing Speed Index (raw score, scaled score, $z$-value), Trail Making Test form A (raw score [in seconds], scaled score, $z$-value), Trail Making Test form B (raw score [in seconds], scaled score, $z$-value), Beck Depression Inventory-II (score).

\section{Experience}

Date of operation (years since January 1, 2003).

\section{In-Hospital Clinical Neurologic Event and Postoperative Imaging Study and Neurocognitive Decline}

Clinical stroke, visual central nervous system symptoms, peripheral twitching, seizure, delirium, brain imaging changes, neurocognitive decline, permanent stroke or stroke diagnosed from MRI, brain imaging changes or permanent stroke, or stroke diagnosed from MRI. 


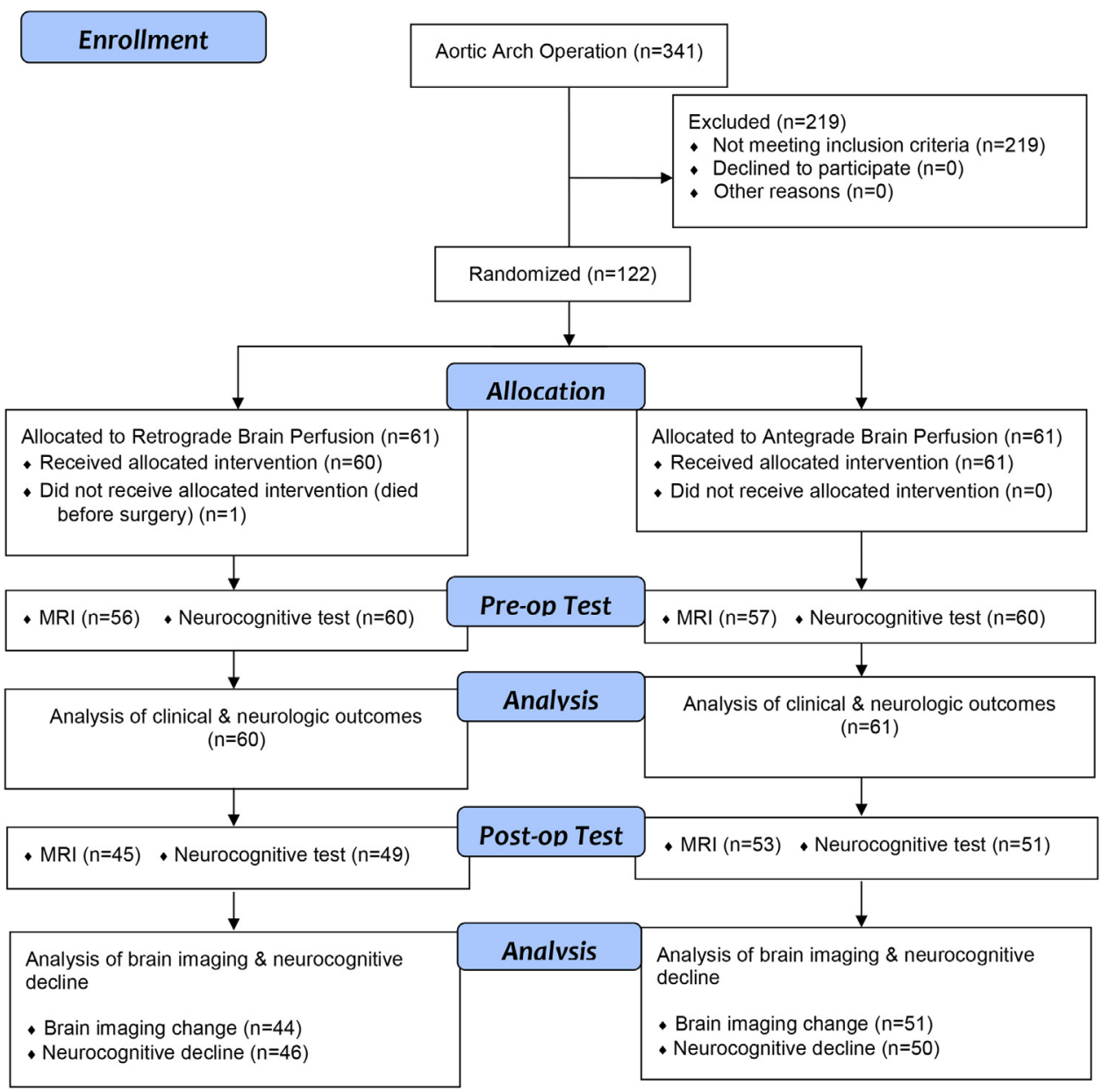

FIGURE E1. CONSORT flow diagram for trial. MRI, Magnetic resonance imaging.

TABLE E1. Intraoperative variables overall and by brain perfusion method

\begin{tabular}{|c|c|c|c|c|c|c|}
\hline \multirow[b]{3}{*}{ Variable } & \multirow{2}{*}{\multicolumn{2}{|c|}{ Overall $(\mathbf{n}=\mathbf{1 2 1})$}} & \multicolumn{4}{|c|}{ Brain perfusion method } \\
\hline & & & \multicolumn{2}{|r|}{ Retrograde $(n=60)$} & \multicolumn{2}{|r|}{ Antegrade $(n=61)$} \\
\hline & $\mathbf{n}^{*}$ & No. $(\%)$ or mean \pm SD & $\mathbf{n} *$ & No. $(\%)$ or mean \pm SD & $\mathbf{n}^{*}$ & No. $(\%)$ or mean \pm SD \\
\hline \multicolumn{7}{|l|}{ Temperature $\left({ }^{\circ} \mathrm{C}\right)$} \\
\hline Nasopharyngeal, low & 113 & $18 \pm 2.9$ & 57 & $18 \pm 1.8$ & 56 & $18 \pm 3.8$ \\
\hline Bladder, low & 120 & $25 \pm 3.7$ & 60 & $25 \pm 3.9$ & 60 & $25 \pm 3.4$ \\
\hline Bladder, high & 120 & $35 \pm 1.3$ & 60 & $35 \pm 1.0$ & 60 & $35 \pm 1.5$ \\
\hline Venous blood, high & 121 & $36 \pm 0.5$ & 60 & $36 \pm 0.53$ & 61 & $36 \pm 0.47$ \\
\hline \multicolumn{7}{|l|}{ Support } \\
\hline Cardiopulmonary bypass time (min) & 121 & $118 \pm 33$ & 60 & $118 \pm 36$ & 61 & $119 \pm 29$ \\
\hline Pump time (min) $\dagger$ & 120 & $92 \pm 32$ & 59 & $91 \pm 34$ & 61 & $93 \pm 30$ \\
\hline Aortic clamp/balloon occlusion used & 121 & $115(95)$ & 60 & $57(95)$ & 61 & $58(95)$ \\
\hline Myocardial ischemic time (min) & 121 & $67 \pm 35$ & 60 & $69 \pm 36$ & 61 & $65 \pm 34$ \\
\hline Blood gas $\mathrm{pH}$ before circulatory arrest & 116 & $7.4 \pm 0.11$ & 58 & $7.4 \pm 0.10$ & 58 & $7.4 \pm 0.12$ \\
\hline Circulatory arrest time (min) & 121 & $27 \pm 13$ & 60 & $26 \pm 12$ & 61 & $31 \pm 14$ \\
\hline Duration blood pressure $<50(\mathrm{~min})$ & 116 & $0 / 6.0 / 33 \ddagger$ & 58 & $0 / 0 / 31 \ddagger$ & 58 & $0 / 13 / 39$ \\
\hline Warming time (min) & 120 & $59 \pm 20$ & 60 & $61 \pm 20$ & 60 & $58 \pm 19$ \\
\hline
\end{tabular}

$S D$, Standard deviation. *Patients with data available. $\dagger$ Excludes cardiac arrest time. $\ddagger 15$ th/50th/85th percentiles. 
TABLE E2. Ninety percent reliable change interval based on $z$-value

Test

Change interval

Hopkins Verbal Learning Test-revised

\begin{tabular}{ll} 
Trial 1 & $(-1.26$ to 1.18$)$ \\
Total & $(-1.58$ to 1.38$)$ \\
Delayed recall & $(-1.77$ to 1.75$)$ \\
Discrimination index & $(-2.41$ to 2.35$)$ \\
Controlled Oral Word Association Test: FAS & $(-1.08$ to 1.16$)$ \\
Boston Naming Test: Total & $(-0.97$ to 1.17$)$ \\
Pegs & \\
Dominant hand & $(-1.25$ to 1.37$)$ \\
Nondominant hand & $(-0.80$ to 1.14$)$ \\
Digit Symbol Test & $(-0.77$ to 1.03$)$ \\
Symbol search & $(-0.96$ to 1.18$)$ \\
Processing speed index & $(-0.71$ to 0.95$)$ \\
Trail Making Test: Part A & $(-1.12$ to 1.16$)$ \\
Trail Making Test: Part B & $(-1.27$ to 1.37$)$ \\
\hline
\end{tabular}

TABLE E3. Neurocognitive test results expressed as $z$-value and reliable change: Retrograde brain perfusion

\begin{tabular}{|c|c|c|c|c|c|c|c|c|c|}
\hline \multirow[b]{2}{*}{ Test } & \multicolumn{2}{|c|}{ Preoperative result } & \multicolumn{2}{|c|}{ Postoperative result } & \multirow[b]{2}{*}{ No. of paired tests } & \multirow[b]{2}{*}{$P$ value } & \multicolumn{3}{|c|}{$\begin{array}{c}\text { Reliable change } \\
\text { No. }(\%) \\
\end{array}$} \\
\hline & $\mathbf{n}^{*}$ & Mean \pm SD & n* & Mean \pm SD & & & Decline & No change & Improvement \\
\hline \multicolumn{10}{|c|}{ Hopkins Verbal Learning Test $\dagger$} \\
\hline Trial 1 & 60 & $0.97 \pm 0.67$ & 48 & $0.90 \pm 0.56$ & 48 & .7 & $1(2.1)$ & $44(92)$ & $3(6.3)$ \\
\hline Total & 60 & $1.12 \pm 0.89$ & 48 & $1.00 \pm 0.81$ & 48 & .5 & $3(6.3)$ & $43(90)$ & $2(4.2)$ \\
\hline Delayed recall & 60 & $1.15 \pm 1.04$ & 48 & $1.11 \pm 1.02$ & 48 & .8 & $3(6.3)$ & $44(92)$ & $1(2.1)$ \\
\hline Discrimination index & 59 & $0.96 \pm 0.84$ & 48 & $0.92 \pm 0.86$ & 47 & $>.9$ & $0(0)$ & $47(100)$ & $0(0)$ \\
\hline COWA & 59 & $0.89 \pm 0.63$ & 47 & $0.95 \pm 0.68$ & 46 & .3 & $3(6.5)$ & $40(87)$ & $3(6.5)$ \\
\hline Boston Naming Test & 60 & $0.21 \pm 0.80$ & 46 & $0.35 \pm 0.93$ & 46 & .3 & $2(4.3)$ & $44(96)$ & $0(0)$ \\
\hline \multicolumn{10}{|l|}{ Grooved Pegboard Test } \\
\hline Dominant hand & 60 & $1.03 \pm 0.85$ & 47 & $1.16 \pm 0.75$ & 47 & .8 & $3(6.4)$ & $39(83)$ & $5(11)$ \\
\hline Nondominant hand & 57 & $0.76 \pm 0.67$ & 47 & $1.09 \pm 0.65$ & 44 & .04 & $3(6.8)$ & $37(84)$ & $4(9.1)$ \\
\hline Digit Symbol Test & 60 & $0.73 \pm 0.55$ & 49 & $0.76 \pm 0.58$ & 49 & .5 & $3(6.1)$ & $45(92)$ & $1(2.0)$ \\
\hline Symbol search & 59 & $0.67 \pm 0.65$ & 49 & $0.71 \pm 0.58$ & 49 & $>.9$ & $4(8.2)$ & $44(90)$ & $1(2.0)$ \\
\hline Processing speed index & 59 & $0.75 \pm 0.61$ & 49 & $0.79 \pm 0.56$ & 49 & .4 & $3(6.1)$ & $45(92)$ & $1(2.0)$ \\
\hline \multicolumn{10}{|l|}{ Trail Making Test } \\
\hline Form A & 60 & $0.86 \pm 0.59$ & 49 & $0.92 \pm 0.72$ & 49 & .5 & $2(4.1)$ & $41(84)$ & $6(12)$ \\
\hline Form B & 60 & $0.94 \pm 0.71$ & 48 & $1.00 \pm 0.69$ & 48 & .8 & $2(4.2)$ & $44(92)$ & $2(4.2)$ \\
\hline
\end{tabular}

COWA, Controlled Oral Word Association Test; $S D$, standard deviation. *Patients with data available. †Revised. 
TABLE E4. Neurocognitive test results expressed as $z$-value and reliable change: Antegrade brain perfusion

\begin{tabular}{|c|c|c|c|c|c|c|c|c|c|}
\hline \multirow[b]{2}{*}{ Test } & \multicolumn{2}{|c|}{ Preoperative result } & \multicolumn{2}{|c|}{ Postoperative result } & \multirow[b]{2}{*}{ No. of paired tests } & \multirow[b]{2}{*}{$P$ value } & \multicolumn{3}{|c|}{ Reliable change no. $(\%)$} \\
\hline & $\mathbf{n}^{*}$ & Mean \pm SD & $\mathbf{n}^{*}$ & Mean \pm SD & & & Decline & No change & Improvement \\
\hline \multicolumn{10}{|c|}{ Hopkins Verbal Learning Test $\dagger$} \\
\hline Trial 1 & 60 & $1.1 \pm 0.68$ & 51 & $1.06 \pm 0.65$ & 51 & .7 & $2(3.9)$ & $48(94)$ & $1(2.0)$ \\
\hline Total & 60 & $1.41 \pm 0.87$ & 51 & $1.24 \pm 0.86$ & 51 & .4 & $4(7.8)$ & $46(90)$ & $1(2.0)$ \\
\hline Delayed recall & 60 & $1.40 \pm 1.14$ & 51 & $1.27 \pm 1.07$ & 51 & .9 & $2(3.9)$ & $45(88)$ & $4(7.8)$ \\
\hline Discrimination index & 60 & $1.27 \pm 1.38$ & 51 & $1.08 \pm 1.04$ & 51 & .8 & $3(5.9)$ & $46(90)$ & $2(3.9)$ \\
\hline COWA & 59 & $0.94 \pm 0.60$ & 50 & $0.90 \pm 0.66$ & 49 & .8 & $3(6.1)$ & $42(86)$ & $4(8.2)$ \\
\hline Boston Naming Test & 59 & $-0.22 \pm 1.14$ & 51 & $-0.13 \pm 1.71$ & 50 & .5 & $2(4.0)$ & $45(90)$ & $3(6.0)$ \\
\hline \multicolumn{10}{|l|}{ Grooved Pegboard Test } \\
\hline Dominant hand & 60 & $0.93 \pm 0.67$ & 51 & $1.00 \pm 0.81$ & 51 & .5 & $1(2.0)$ & $47(92)$ & $3(5.9)$ \\
\hline Nondominant hand & 59 & $0.76 \pm 0.55$ & 50 & $0.90 \pm 0.69$ & 50 & .13 & $4(8.0)$ & $43(86)$ & $3(6.0)$ \\
\hline Digit Symbol Test & 60 & $0.66 \pm 0.53$ & 51 & $0.79 \pm 0.59$ & 51 & .02 & $1(2.0)$ & $45(88)$ & $5(9.8)$ \\
\hline Symbol search & 60 & $0.65 \pm 0.61$ & 51 & $0.83 \pm 0.61$ & 51 & .01 & $1(2.0)$ & $46(90)$ & $4(7.8)$ \\
\hline Processing speed index & 60 & $0.73 \pm 0.51$ & 51 & $0.87 \pm 0.57$ & 51 & .01 & $0(0)$ & $45(88)$ & $6(12)$ \\
\hline \multicolumn{10}{|l|}{ Trail Making Test } \\
\hline Form A & 60 & $0.79 \pm 0.58$ & 51 & $0.73 \pm 0.61$ & 51 & .6 & $3(5.9)$ & $45(88)$ & $3(5.9)$ \\
\hline Form B & 59 & $0.81 \pm 0.70$ & 51 & $0.92 \pm 0.73$ & 50 & .5 & $3(6.0)$ & $45(90)$ & $2(4.0)$ \\
\hline
\end{tabular}

COWA, Controlled Oral Word Association Test; $S D$, standard deviation. *Patients with data available. $\dagger$ Revised.

TABLE E5. Neurologic outcomes in patients with and without prior stroke

\begin{tabular}{|c|c|c|c|c|c|}
\hline \multirow[b]{3}{*}{ Outcome } & \multicolumn{4}{|c|}{ Prior stroke } & \multirow[b]{3}{*}{$P$ value } \\
\hline & \multicolumn{2}{|r|}{ No } & \multicolumn{2}{|r|}{ Yes } & \\
\hline & n* & No. $(\%)$ & $\mathbf{n}^{*}$ & No. $(\%)$ & \\
\hline Stroke & 105 & $0(0)$ & 16 & $1(6.3)$ & .13 \\
\hline Visual & 105 & $1(0.95)$ & 16 & $1(6.3)$ & .3 \\
\hline Seizure & 105 & $1(0.95)$ & 16 & $0(0)$ & $>.9$ \\
\hline Twitching & 105 & $2(1.9)$ & 16 & $1(6.3)$ & .4 \\
\hline Delirium & 105 & $1(0.95)$ & 16 & $1(6.3)$ & .3 \\
\hline Brain imaging changes & 86 & $12(14)$ & 9 & $2(22)$ & .6 \\
\hline Neurocognitive decline & 86 & $17(20)$ & 10 & $0(0)$ & .2 \\
\hline Composite event & 83 & $27(33)$ & 7 & $2(29)$ & $>.9$ \\
\hline
\end{tabular}

*Patients with data available. †If frequency $<5$, Fisher's exact test was used to compare the groups, and corresponding $P$ value is given. 
TABLE E6. Matrix of postoperative clinical events, brain imaging, and neurocognitive outcomes: Retrograde brain perfusion

\begin{tabular}{|c|c|c|c|c|c|c|c|c|}
\hline \multirow[b]{2}{*}{ Pattern } & \multirow[b]{2}{*}{ No. of patients } & \multicolumn{5}{|c|}{ Clinical } & \multirow[b]{2}{*}{ Brain imaging changes } & \multirow[b]{2}{*}{ Neurocognitive decline } \\
\hline & & Stroke & Visual & Seizure & Twitching & Delirium & & \\
\hline 1 & 1 & Yes & No & No & No & No & Yes & N/A \\
\hline 2 & 1 & No & Yes & No & No & No & Yes & No \\
\hline 3 & 1 & No & Yes & No & No & No & Yes & Yes \\
\hline 4 & 0 & No & No & Yes & No & No & No & No \\
\hline 5 & 0 & No & No & No & Yes & No & No & N/A \\
\hline 6 & 0 & No & No & No & Yes & No & No & No \\
\hline 7 & 1 & No & No & No & No & Yes & N/A & N/A \\
\hline 8 & 0 & No & No & No & No & Yes & No & No \\
\hline 9 & 7 & No & No & No & No & No & N/A & N/A \\
\hline 10 & 5 & No & No & No & No & No & N/A & No \\
\hline 11 & 3 & No & No & No & No & No & N/A & Yes \\
\hline 12 & 4 & No & No & No & No & No & No & N/A \\
\hline 13 & 1 & No & No & No & No & No & Yes & N/A \\
\hline 14 & 4 & No & No & No & No & No & Yes & No \\
\hline 15 & 1 & No & No & No & No & No & Yes & Yes \\
\hline 16 & 26 & No & No & No & No & No & No & No \\
\hline 17 & 5 & No & No & No & No & No & No & Yes \\
\hline
\end{tabular}

TABLE E7. Matrix of postoperative clinical events, brain imaging, and neurocognitive outcomes: Antegrade brain perfusion

\begin{tabular}{|c|c|c|c|c|c|c|c|c|}
\hline \multirow[b]{2}{*}{ Pattern } & \multirow[b]{2}{*}{ No. of patients } & \multicolumn{5}{|c|}{ Clinical } & \multirow[b]{2}{*}{ Brain imaging changes } & \multirow[b]{2}{*}{ Neurocognitive declin } \\
\hline & & Stroke & Visual & Seizure & Twitching & Delirium & & \\
\hline 1 & 0 & Yes & No & No & No & No & Yes & N/A \\
\hline 2 & 0 & No & Yes & No & No & No & Yes & No \\
\hline 3 & 0 & No & Yes & No & No & No & Yes & Yes \\
\hline 4 & 1 & No & No & Yes & No & No & No & No \\
\hline 5 & 1 & No & No & No & Yes & No & No & N/A \\
\hline 6 & 2 & No & No & No & Yes & No & No & No \\
\hline 7 & 0 & No & No & No & No & Yes & N/A & N/A \\
\hline 8 & 1 & No & No & No & No & Yes & No & No \\
\hline 9 & 4 & No & No & No & No & No & N/A & N/A \\
\hline 10 & 5 & No & No & No & No & No & N/A & No \\
\hline 11 & 1 & No & No & No & No & No & N/A & Yes \\
\hline 12 & 4 & No & No & No & No & No & No & N/A \\
\hline 13 & 2 & No & No & No & No & No & Yes & N/A \\
\hline 14 & 3 & No & No & No & No & No & Yes & No \\
\hline 15 & 0 & No & No & No & No & No & Yes & Yes \\
\hline 16 & 31 & No & No & No & No & No & No & No \\
\hline 17 & 6 & No & No & No & No & No & No & Yes \\
\hline
\end{tabular}


TABLE E8. Postoperative in-hospital complications

\begin{tabular}{|c|c|c|c|c|c|c|c|}
\hline \multirow[b]{3}{*}{ Complication } & & & \multicolumn{5}{|c|}{ Brain perfusion method } \\
\hline & \multicolumn{2}{|c|}{ Overall $(n=121)$} & \multicolumn{2}{|c|}{ Retrograde $(n=60)$} & \multicolumn{2}{|c|}{ Antegrade $(n=61)$} & \multirow[b]{2}{*}{$P$ value } \\
\hline & $\mathbf{n}^{*}$ & No. $(\%)$ & $\mathbf{n}^{*}$ & No. $(\%)$ & $\mathbf{n}^{*}$ & No. $(\%)$ & \\
\hline Atrial fibrillation or flutter & 119 & $41(34)$ & 59 & $22(37)$ & 60 & $19(32)$ & .5 \\
\hline Prolonged ventilation (>24 h) & 120 & $36(30)$ & 60 & $16(27)$ & 60 & $20(33)$ & .4 \\
\hline Reoperation for bleeding or tamponade & 121 & $6(5.0)$ & 60 & $2(3.3)$ & 61 & $4(6.6)$ & .4 \\
\hline Renal failure requiring dialysis & 121 & $5(4.1)$ & 60 & $3(5.0)$ & 61 & $2(3.3)$ & .6 \\
\hline Septicemia & 121 & $2(1.7)$ & 60 & $0(0)$ & 61 & $2(3.3)$ & .16 \\
\hline Deep sternal wound infection & 121 & $1(0.83)$ & 60 & $0(0)$ & 61 & $1(1.6)$ & .3 \\
\hline Perioperative myocardial infarction & 121 & $1(0.83)$ & 60 & $1(1.7)$ & 61 & $0(0)$ & .3 \\
\hline
\end{tabular}

*Patients with data available.

TABLE E9. Frequencies of imputed components and of the resulting composite event in the imputed complete data sets using a cutoff of 0.5

\begin{tabular}{lcccccccccc}
\hline \multicolumn{1}{c}{ Imputation } & $\mathbf{1}$ & $\mathbf{2}$ & $\mathbf{3}$ & $\mathbf{4}$ & $\mathbf{5}$ & $\mathbf{6}$ & $\mathbf{7}$ & $\mathbf{8}$ & $\mathbf{9}$ & $\mathbf{1 0}$ \\
\hline Brain imaging changes & 19 & 19 & 19 & 18 & 20 & 21 & 21 & 21 & 21 & 21 \\
Neurologic decline & 20 & 20 & 20 & 20 & 19 & 19 & 20 & 20 & 20 & 20 \\
Composite event & 37 & 37 & 37 & 36 & 36 & 37 & 38 & 38 & 38 & 38 \\
\hline
\end{tabular}

